OPEN ACCESS

Edited by:

Sara Pedron,

University of llinois at Urbana-

Champaign, United States

Reviewed by:

Stephanie Michelle Willerth,

University of Victoria, Canada

Stephanie K. Seidlits,

University of California, Los Angeles,

United States

Francesca Taraballi,

Houston Methodist Research

Institute, United States

*Correspondence:

Edi Meco

em4zv@virginia.edu;

Kyle J. Lampe

lampe@virginia.edu

Specialty section:

This article was submitted to

Biomaterials,

a section of the journal

Frontiers in Materials

Received: 28 October 2017

Accepted: 10 January 2018

Published: 01 February 2018

Citation:

Meco E and Lampe KJ (2018) Microscale Architecture in Biomaterial Scaffolds for Spatial Control of Neural

Cell Behavior.

Front. Mater. 5:2.

doi: 10.3389/fmats.2018.00002

\section{Microscale Architecture in Biomaterial Scaffolds for Spatial Control of Neural Cell Behavior}

\author{
Edi Meco* and Kyle J. Lampe* \\ Department of Chemical Engineering, University of Virginia, Charlottesville, VA, United States
}

Biomaterial scaffolds mimic aspects of the native central nervous system (CNS) extracellular matrix (ECM) and have been extensively utilized to influence neural cell (NC) behavior in in vitro and in vivo settings. These biomimetic scaffolds support NC cultures, can direct the differentiation of NCs, and have recapitulated some native NC behavior in an in vitro setting. However, NC transplant therapies and treatments used in animal models of CNS disease and injury have not fully restored functionality. The observed lack of functional recovery occurs despite improvements in transplanted NC viability when incorporating biomaterial scaffolds and the potential of NC to replace damaged native cells. The behavior of NCs within biomaterial scaffolds must be directed in order to improve the efficacy of transplant therapies and treatments. Biomaterial scaffold topography and imbedded bioactive cues, designed the microscale level, can alter NC phenotype, direct migration, and differentiation. Microscale patterning in biomaterial scaffolds for spatial control of NC behavior has enhanced the capabilities of in vitro models to capture properties of the native CNS tissue ECM. Patterning techniques such as lithography, electrospinning and three-dimensional (3D) bioprinting can be employed to design the microscale architecture of biomaterial scaffolds. Here, the progress and challenges of the prevalent biomaterial patterning techniques of lithography, electrospinning, and 3D bioprinting are reported. This review analyzes $\mathrm{NC}$ behavioral response to specific microscale topographical patterns and spatially organized bioactive cues.

\section{Keywords: lithography, three-dimensional bioprinting, electrospinning, biomaterials, neural stem cells}

\section{INTRODUCTION}

\section{Tissue Damage in the Central Nervous System (CNS)}

Tissue damage in the CNS caused by injury and disease cannot be fully repaired through endogenous healing mechanisms. For therapeutic treatments of CNS injury and disease to be successful they need to address a variety of challenges that are specific to the individual injury or disease, but can be broadly defined as replacing dead neural cells (NCs), remodeling the extracellular matrix (ECM) to a healthy state, and restoring nervous system functionality. In the autoimmune disease multiple sclerosis (MS), for instance, immune cells infiltrate the CNS and cause demyelination of neuronal axons (Correale et al., 2015). In the aftermath of this demyelination, the axons deteriorate and neuronal death occurs, leading to reactive astrogliosis (Correale et al., 2015). During reactive astrogliosis astrocytes form inhibitory glial scars around the demyelinated lesion and prevent both remyelination 
of damaged axons and axonal regeneration (Correale et al., 2015). The therapeutic needs for successful tissue regeneration of this particular disease are to replace dead neurons and oligodendrocytes, remodel the inhibitory scar tissue to allow for infiltration of endogenous cells, and prevent further immune cell infiltration into the CNS. A related sequence of events occurs in ischemic stroke, where the blood supply to the CNS is blocked, leading to hypoxia. Unlike MS, where preventative medicine is used to inhibit immune cell infiltration and mitigate tissue damage, tissue damage in ischemic stroke is difficult to prevent because it only takes approximately $4 \mathrm{~min}$ for the adenosine triphosphate (ATP) concentration to be fully depleted (Krause et al., 1988). Most of the cell death occurs during reperfusion, when blood flow returns to the CNS and the oxygen concentration suddenly elevates (White et al., 2000). Re-established metabolism of oxygen causes the overproduction of reactive oxygen species (ROS), leading to lipid peroxidation of unsaturated fatty acids on the cell membranes, increasing their permeability, and causing apoptosis (White et al., 2000; Lipinski, 2011). This is followed by reactive astrogliosis and formation of a glial scar around the original oxygen deficient region (Huang et al., 2014). Successful therapeutic treatment of damaged tissue after ischemic strike would replace dead NCs and remodel the inhibitory scar tissue to allow for infiltration of endogenous cells. There is a significant difference in the types of cells that need to be replaced when comparing ischemic stroke, which indiscriminately leads to NC death within a region, to diseases like MS which targets specific cell types. Unlike MS and ischemic stroke, tissue damage in the form of cell necrosis occurs immediately after a mechanical insult in spinal cord (SC) contusion injury (Yuan and He, 2013). Cell debris and ROS cause cytotoxicity in the microenvironment, and subsequently apoptosis of surrounding cells (Macaya and Spector, 2012). Over the course of several days to months after the initial injury, reactive astrocytes, in conjunction with infiltrating periphery cells in some instances, form glial scar tissue in the area of the mechanical insult (White et al., 2000; Macaya and Spector, 2012). Treatment of CNS tissue damaged by SC contusion injury requires ECM remodeling to remove the cytotoxic cell debris and excess ROS before cells in the injury lesion can be replaced. The mechanisms of NC death are different in each case, but the requirements for effective treatments can be broadly defined as remodeling the ECM to remove cytotoxic and inhibitory aspects, replacing damaged cells, and recovering functionality.

\section{Reparative Effects of Endogenous NCs}

Interest in developing NC transplant therapies to treat tissue damage caused by CNS injury and disease is derived from evidence that endogenous NCs have reparative effects on damaged tissue (Cooke et al., 2010). After ischemic stroke in the rat stratium via middle cerebral artery occlusion (MCAO), new neurons derived from proliferating neural precursor cells (NPCs) in the subventricular zone (SVZ) were observed (Arvidsson et al., 2002). Similar results occurred in mice with an ipsilateral cerebral cortex infarction induced by MCAO, where the number of Nestin-positive cells in the ischemic region increased (Nakagomi et al., 2009). When extracted and cultured, the Nestin-positive cells from the ischemic region displayed neural stem cell (NSC) properties in vitro (Nakagomi et al., 2009). NSC populations, derived from ependymal cells, were also discovered in mouse SC after a transverse dorsal funiculus incision (Barnabé-Heider et al., 2010). These findings have led researchers to transplant NCs as therapies to heal CNS tissue damage in both the brain and SC. However, most of the NCs transplanted to the injury environment do not survive and cells that do survive do not display integrative behavior. Only $2-4.5 \%$ of mouse NPCs transplanted into rats after traumatic brain injury survived $24 \mathrm{~h}$ after the transplant (Bakshi et al., 2005). In addition, the caspase activity of transplanted NPCs that survived was higher in injured rats than sham surgery controls, indicating that the population of cells that survived was apoptotic in the CNS injury environment (Bakshi et al., 2005). While transplanted cell viability is higher if the transplant is performed 1 week postinjury (Hill et al., 2006; Walker et al., 2015), NCs alone do not restore functionality to preinjury baselines.

It is important to highlight distinctions between NC populations used to research in vitro and in vivo models because there is a wide range of capabilities of each cell line. Mature NCs such as neurons do not contain the capacity to self-renew or further differentiate and are typically not used because in vitro cell cultures cannot be maintained. NSCs can self-renew indefinitely and exhibit multipotent differentiation, while NPCs and neural progenitor cells have a limited capacity to self-renew and have a restricted capacity to differentiate (Seaberg and Van Der Kooy, 2003). Typically NPCs display unipotent differentiation behavior while neural progenitor cells maintain multipotent differentiation, but these descriptions are not rigid rules so overlap between NPCs and neural progenitor cells does exist in literature. Neural precursor and progenitor cells are preferred over NSCs for transplant therapies because their differentiation is more controlled, resulting in less heterogeneous cell cultures. Many NC lines are derived from neuronal tumors because these cell lines are immortalized and are relatively easy to grow and maintain in in vitro cell cultures (Gordon et al., 2013). The capabilities and limitations of NC lines are discussed by other reviews (Seaberg and Van Der Kooy, 2003; Dell'Albani, 2008; Murry and Keller, 2008; Politis and Lindvall, 2012; Gordon et al., 2013). Here, we will focus on the microscale architecture design of biomaterial scaffolds and how NCs respond to that architecture.

\section{Bulk Biomaterials for Tissue Repair}

Biomaterial scaffolds are designed to be biocompatible and influence cell behavior, making them promising tools for developing CNS tissue treatment therapies. Implantation of biomaterials into CNS injury lesions has helped improve cell infiltration and functional recovery (Shrestha et al., 2014). A collagen and PuraMatrix hydrogel transplanted into a 5-mm gap thoracic (T9-T11) rat SC transection increased animal Basso, Beattie, and Bresnahan (BBB) scores over phosphatebuffered saline (PBS) controls 4 months postsurgery (Kaneko et al., 2015). Neuronal and astrocyte infiltration into the injury lesion increased in animals with the collagen and PuraMatrix biomaterial scaffold. A similar endogenous cell response occurred when implanting a fibronectin-based scaffold into 2-mm gap thoracic (T7-T9) rat SC transection (King et al., 
2003, 2006). Axons infiltrated the fibronectin scaffold and were myelinated by Schwann cells 4 weeks postinjury (King et al., 2003). Animals with the fibronectin scaffold also had early and aggressive macrophage infiltration into the lesion site, which was speculated to provide trophic support for axon infiltration (King et al., 2006). Biomaterial scaffolds have also been utilized to transplant NCs into damaged CNS tissue. NCs transplanted with biomaterial scaffolds have improved posttransplant viability and improve functional recovery over the standalone scaffold and NC transplantations. Improved functional recovery from a 10-mm gap thoracic (T9-T10) SC hemisection injury on African green monkeys was observed when implanting human NSCs seeded on a poly(lactide-co-glycolide) (PLGA) scaffold (Pritchard et al., 2010). Implanting the PLGA scaffold improved the left hindlimb neuromotor score 44 days postinjury over notreatment controls, and incorporating human NSCs into the PLGA scaffold led to further improvement. Similar results were found when human NSCs in a fibrin scaffold were transplanted into a complete $2-\mathrm{mm}$ long thoracic (T3) rat SC transection, where the inclusion of human NSCs with the fibrin scaffold improved hindlimb locomotion $\mathrm{BBB}$ scores 8 weeks postinjury over untreated controls ( $\mathrm{Lu}$ et al., 2012b). While implanting NCs with biomaterial scaffolds has been shown to promote CNS tissue recovery in animal models, regeneration is still limited and functionality cannot be restored to the preinjury state. Biomaterial scaffolds need to be designed with greater control over NC behavior to improve the efficacy of transplant therapies.

\section{Microscale Architecture in Biomaterials}

Current biomaterial design strategies are focused on controlling NC behavior at the microscale level (less than $1 \mathrm{~mm}$ in size). The goals for controlling microscale architecture within biomaterial scaffolds are to direct $\mathrm{NC}$ behavior toward clinical therapy needs and to investigate how NCs interact with the ECM. The microscale architecture within biomaterials has a significant impact on cell behavior and differentiation (Gunther et al., 2015; Lynam et al., 2015; Dye et al., 2016). Chitosanbased hydrogels were fragmented into varying microscale sizes (Figure 1A) and incorporated into thoracic (T8-T9) rat SC bilateral dorsal hemisection injury model (Chedly et al., 2017). The hydrogel transplants improved endogenous cell infiltration into the injury lesion (Figures 1B-D) and the degree of cell infiltration depended largely on the chitosan fragment size. Hydrogels with $20-\mu \mathrm{m}$ chitosan fragments (Figure 1D) led to robust endogenous cell infiltration while hydrogels with 150- $\mu \mathrm{m}$ chitosan fragments (Figure 1C) had limited cell infiltration 4 weeks postinjury. In addition, scaffolds with $150-\mu \mathrm{m}$ fragments did not have infiltration of axons and few glial cells, while scaffolds with $20-\mu \mathrm{m}$ fragments had robust infiltration of both. While the microstructure alterations were not designed to direct endogenous NC behavior in a specific way, these results illustrate the significance scaffold microstructure on NC behavior for clinical applications. For SC injury, a clinical goal is to guide axons through the injury lesion, which was only achieved by the hydrogel with $20-\mu \mathrm{m}$ fragments. These results provide motivation for exploring techniques that allow greater control over material architecture and cell positioning to guide tissue growth. The prevailing techniques used to design microscale architecture in biomaterial scaffolds for influence over NC behavior are lithography, electrospinning, and threedimensional (3D) bioprinting (Figure 2).

\section{LITHOGRAPHY}

\section{Lithographic Techniques}

Several lithographic techniques are used to pattern biomaterial scaffolds: photolithography, soft lithography, stereolithography, and two-photon lithography (sometimes referred to by the more general term multiphoton lithography in literature). Photolithography refers to techniques that pattern a photoresist onto a substrate using a photomask and a light source, usually in the ultraviolet (UV) wavelength range (Jang et al., 2016). The substrate is the biomaterial housing and the photoresist is a material that is chemically responsive to the presence of light within a specific wavelength range. A photomask is used to create a pattern by shielding parts of the photoresist material from the light source. The process can be repeated to create multiple layers with differing patterns. Since most of the processing is cytotoxic, for biological research the photoresist is patterned, washed, and cells are seeded on top of the material afterward. Stereolithography is similar to photolithography except the photoresist material is replaced with a photocurable polymer resin or solution (Wang et al., 2017). The polymer resin or solution is solidified in the presence of a light source. Patterns are made either by using a photomask or a mobile laser light source. Soft lithography is a technique used in conjunction with photolithography. First a patterned stamp or mold is made from materials such as polydimethylsiloxane (PDMS) using photolithography (Turunen et al., 2013). The patterned stamp is then placed on the substrate and the voids created by the stamp's pattern are filled with a biomaterial of interest, called ink. The benefits of patterning using soft lithography are that the ink solidification chemistry does not need to be light based and the processing can be biocompatible. Two-photon lithography is an extension of photolithography and stereolithography because it is used to pattern the same materials, either a photoresist or photocurable polymer, with a different light source. The UV light source is replaced with a femtosecond pulsed infrared (IR) laser, such as titanium:sapphire lasers, going through an objective lens to focus the beam. The laser wavelength is twice that of the maximum absorbance wavelength of UV light-based chemistry; therefore, two of these lower energy photons are required to generate the same free radical (Ciuciu and Cywiński, 2014). Since the process of absorbing two photons requires a high light intensity, absorbance only occurs at the objective lens focal point, allowing for patterning in the $z$-direction of a UV light responsive material. The advantage of two-photon lithography is the increased resolution in the $z$-direction, which allows for patterning in three dimensions. In contrast, photo- and stereolithography techniques require repeating the processing to create multiple layers for design of 3D structures. Lithography-based techniques have been successfully used to organize biomaterial topography and spatially immobilized ECM materials. 

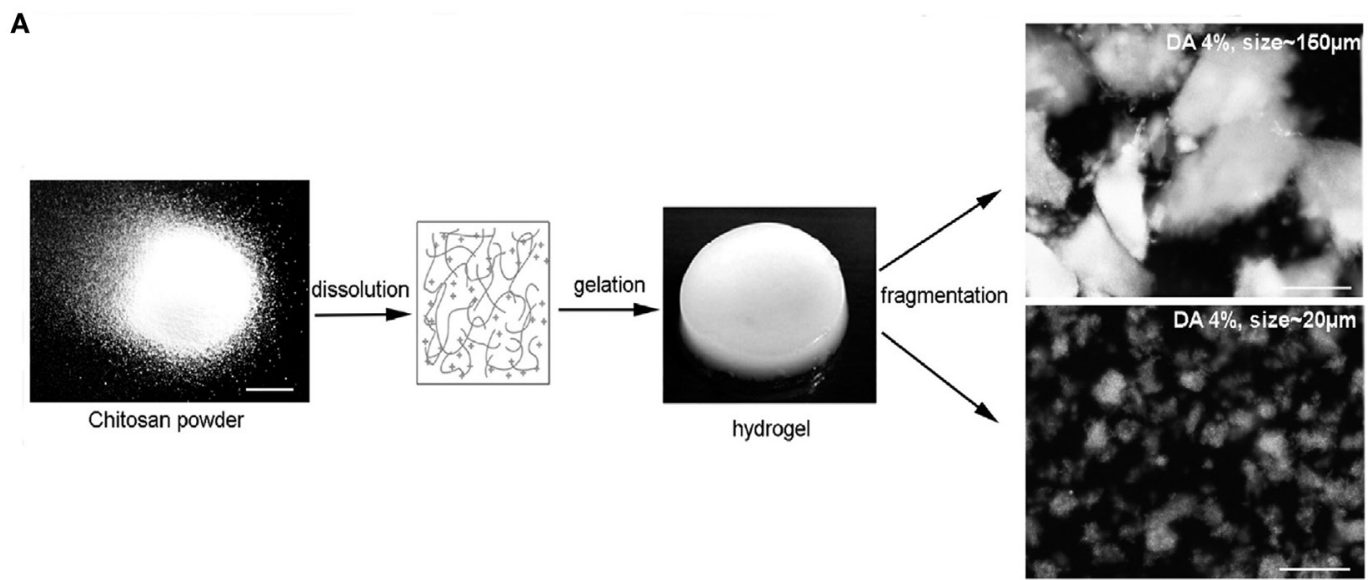

B

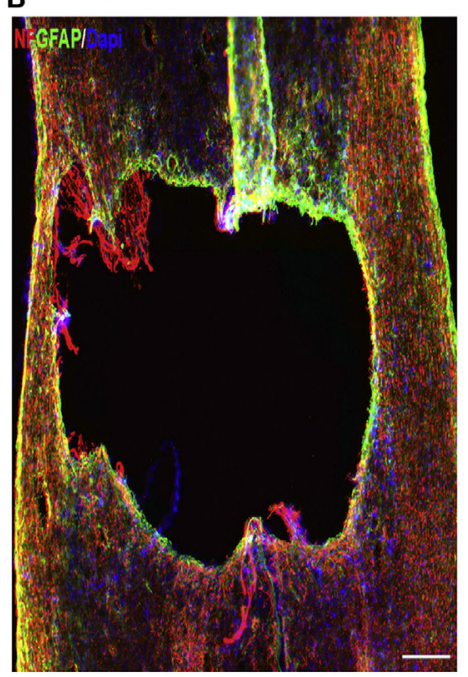

C

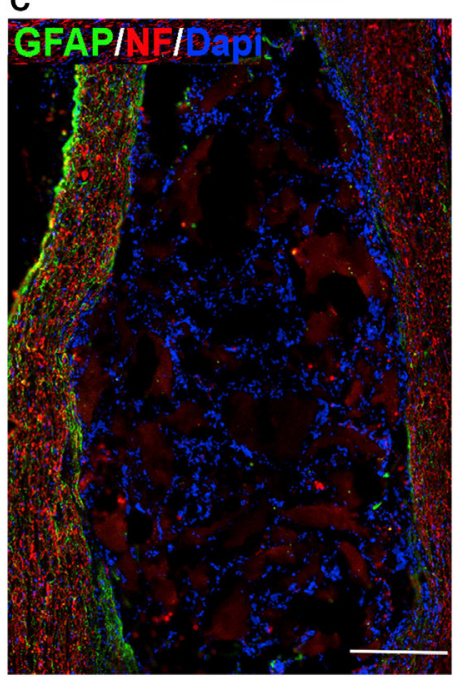

D

injectable physical microgel suspension

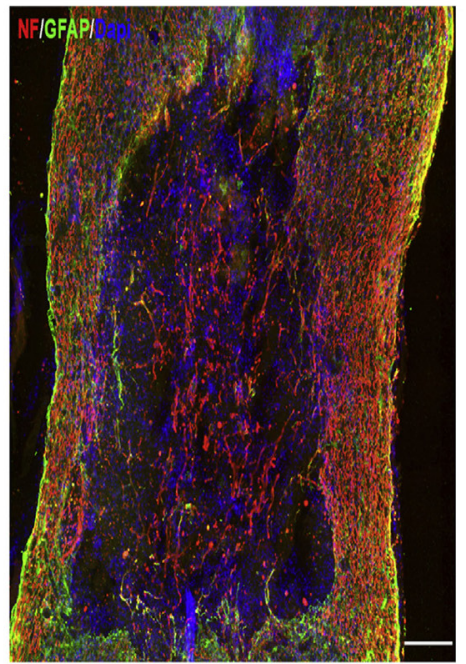

FIGURE 1 | (A) Chitosan solution gelled by contacting with ammonia vapors and then fragmented mechanically into differing average microscale architecture. (B-D) Immunostaining of spinal cord lesion site 4 weeks postinjury/injection for neurofilament (NF), astrocytes (GFAP), and cell nuclei (DAPI). (B) Lesion only, (C) lesion with chitosan-FPHS hydrogel implant with 150- $\mu \mathrm{m}$ average fragment size, and (D) lesion with chitosan-FPHS $20-\mu \mathrm{m}$ average fragment size. Smaller fragments result in greater presence of NF in the lesion site. (B,D) Scale bar $=240 \mu \mathrm{m}$ and (C) Scale bar $=300 \mu \mathrm{m}$. Reproduced with permission of Chedly et al. (2017), Copyright 2017, Elsevier.

\section{Grooves on Scaffold Surfaces}

Lithographic patterning of grooves into biomaterial surfaces has been extensively shown to direct $\mathrm{NC}$ alignment, migration, and differentiation (Dos Reis et al., 2010; Haring et al., 2017). Astrocytes seeded onto a polystyrene mold patterned with channels that were $10-\mu \mathrm{m}$ wide and $3-\mu \mathrm{m}$ deep using photolithography, and subsequently coated with laminin, had elongated process extensions that aligned with the grooves (Recknor et al., 2004). In the non-patterned surface astrocytes had rounded cell bodies and process extensions were not oriented toward a particular direction. Adult hippocampal progenitor cells behaved in a similar manner by aligning processes with patterned channels on a laminin-coated polystyrene scaffold (Oh et al., 2009). In addition, cells seeded onto the patterned scaffold had longer process extensions than those on the non-patterned surface. Differentiation down the neuronal, oligodendrocyte, and astrocyte pathways was similar for cells seeded on both the patterned and non-patterned polystyrene surfaces, indicating that this effect was not caused by a difference in the cell differentiation. The dimensions of the grooves are important for being able to control both differentiation and alignment. Dorsal root ganglion (DRG) cells seeded onto the surface of a coumarin-based biomaterial scaffold that was patterned into square pillars using a modified stereolithography technique did not show robust alignment with the grooves (McCormick et al., 2014). This could be because the grooves were very shallow, with $20 \mathrm{~nm}$ being the largest depth tested. Other studies have investigated the effects of groove width on NC behavior. Adult human NSCs aligned better on smaller, 5 - $\mu \mathrm{m}$ wide, channels than on larger, $60-\mu \mathrm{m}$ wide, channels when seeded onto patterned PDMS channels coated with poly-L-lysine (Béduer et al., 2012). However, more cells seeded on the PDMS scaffold with larger channels differentiated down the neuronal lineage, and had more neurites per cell. Similar results were found when radial glia cells were seeded on polymethylmethacrylate 


\section{D Bioprinting Electrospinning Lithography}

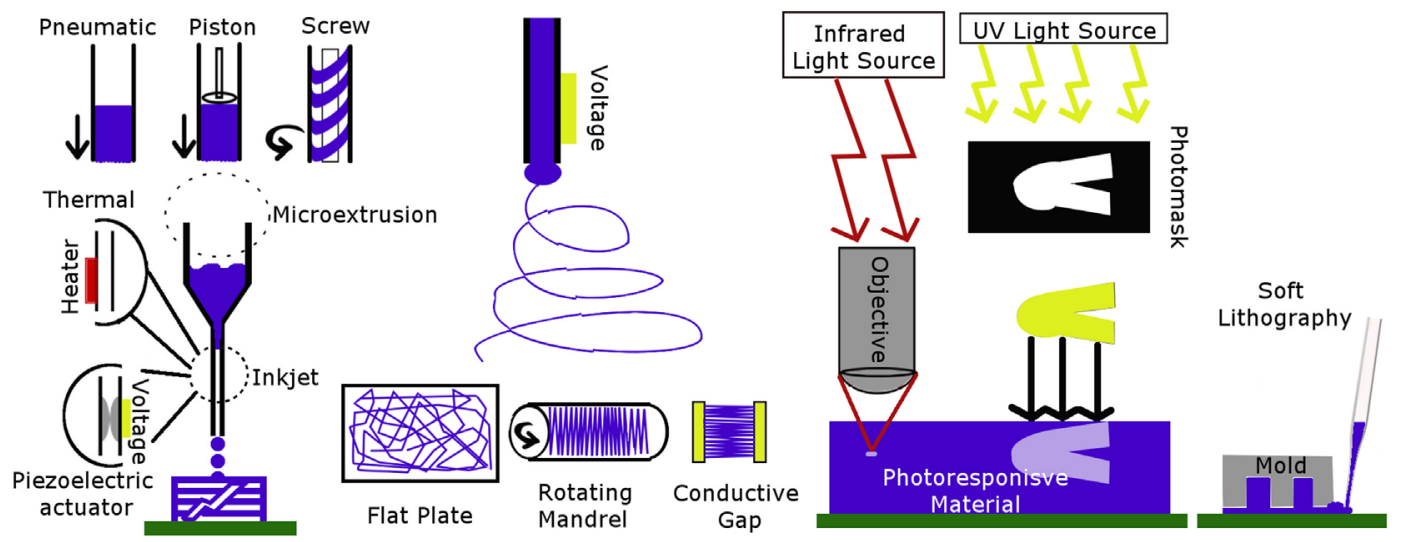

FIGURE 2 | Schematic of 3D bioprinting, electrospinning, and lithography techniques. The type of 3D bioprinter is chosen based on biomaterial rheological properties. Electrospun fibers are patterned by changing the collector. Lithography patterning is done by adjusting light-based techniques or using a mold.

(PMMA) scaffolds patterned with 2- and 10- $\mu$ m wide channels, where cells aligned with the grooves and migrated along the channels (Figure 3) (Mattotti et al., 2012). Patterning channels on the scaffold surface can also affect the behavior of NCs encapsulated within the biomaterial scaffold. Bone marrow-derived stem cells encapsulated in alginate gels with $500-\mu \mathrm{m}$ wide surface microchannels had a fourfold decrease in the glial cell to neuron differentiation ratio when compared to non-patterned hydrogels (Lee et al., 2015). Simulations of oxygen and water transport into the alginate hydrogels indicated that the surface microchannels improved oxygen and water diffusion into the scaffolds. The decrease in the glial cell to neuron differentiation ratio was attributed to better nutrient transport in hydrogels with the surface microchannels. Lithographic patterning can also be used to spatially orient bioactive motifs to direct NC behavior.

\section{Immobilization of Bioactive Motifs}

Lithographic techniques have been developed that can spatially immobilize proteins and ECM molecules onto the polymer network of biomaterial scaffolds. A prevalent theme in the literature is the microscale attachment of adhesive ECM proteins and peptide sequences, such as arginine-glycine-aspartic acid (RGD), which can be used to direct cell migration and the extension of processes (Hahn et al., 2005; Huval et al., 2015). DRG cells seeded on the surface of an agarose scaffold migrated and extended processes into the interior of the scaffold when RGD was immobilized within the agarose scaffold (Luo and Shoichet, 2004a,b). The RGD polypeptide was incorporated into the agarose hydrogel by first functionalizing agarose with 2-nitrobenzyl-protected cysteine. The nitrobenzyl group was cleaved to expose a free thiol group when UV light was shined onto the agarose scaffold. The free thiol group subsequently reacted with a maleimide-modified RGD polypeptide dissolved in the scaffold encompassing solution. RGD attachment onto the agarose scaffold was constrained to cylindrical sections down the $z$-axis by using a photomask with open circles. The versatility of this method was demonstrated by achieving the similar results on a hyaluronic acid (HA)-based scaffold (Musoke-Zawedde and Shoichet, 2006). Adhesive motifs can also be used to direct where NCs attach on biomaterial surfaces. LRM55 astroglioma and primary rat hippocampal neurons seeded onto an acrylamidebased substrate with immobilized fibronectin, laminin, and the IKVAV polypeptide sequence (separately) only attached to the fibronectin, laminin or IKVAV portion of the scaffold (Hynd et al., 2006, 2009). Soft lithography was used to immobilize the adhesive motifs into a crosshatch pattern. Primary rat hippocampal neurons extended processes preferentially along the grid pattern and were found to have functional synapses 10 days post seeding. Similar results were achieved when hybrid mouse neuroblastoma and rat glioma cell cultures were seeded onto a bovine serum albumen (BSA)-modified collagen scaffold surface with immobilized matrigel (Nagamine et al., 2015). Matrigel was immobilized into a crosshatch pattern and cells migrated to the nodes of the matrigel pattern, extended processes along the matrigel grid $15 \mathrm{~h}$ after seeding. Although most research has focused patterning motifs that promote adhesion, the techniques described here are versatile and can be expanded to immobilize other proteins.

The biological activity of immobilized proteins depends on the immobilization process and the specific protein tolerances to the processing environment(s). Photolithography was used to test if the activity of two known axon inhibitor proteins, semaphorin $6 \mathrm{~A}$ and ephrin-B3, was affected by immobilizing them onto an agarose-based hydrogel (Curley et al., 2014; Horn-ranney et al., 2014). The technique involved creating a mold from poly(ethylene glycol) (PEG) designed in the shape of a well attached to a channel that splits into two separate channels. The mold was then filled with 2-nitrobenzyl-protected cysteine-modified-agarose solution, similar to the chemistry described above. Semaphorin $6 \mathrm{~A}$ or ephrin-B3 were immobilized onto one of the two channels by exposing the scaffold to UV light and using a photomask. The second channel did not contain immobilized protein and was 

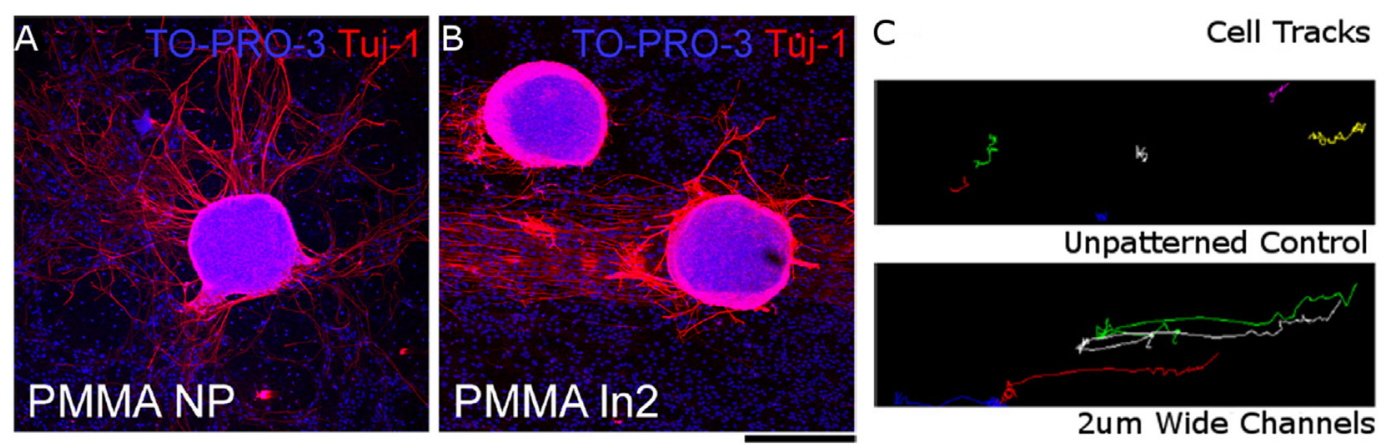

FIGURE 3 | Radial glial cells seeded on (A) unpatterned PMMA surface and (B) PMMA surface with 2- 4 m wide channels. Cell nuclei stained with TO-PRO-S (blue) and neurons stained with beta tubulin III (red). (C) Particle tracks of neuronal trajectories over $3 \mathrm{~h}$ in unpatterned PMMA (top) and PMMA with 2- $\mu$ m wide channels (bottom). Scale bar = $200 \mu \mathrm{m}$. Reproduced with permission of Mattotti et al. (2012), Copyright 2012, Elsevier.

used as a control. DRG explants from the lumbosacral, thoracic, and cervicothoracic regions were placed inside the well and the direction of their process extensions was used to determine if the immobilized proteins maintained biological activity. DRG explants from the lumbosacral region did not extend processes into the channel with immobilized semaphorin 6A, indicating that the immobilized protein was still biologically active. However, processes from thoracic and cervicothoracic DRG explants were not inhibited by semaphorin 6A. In addition, Ephrin-B3 did not inhibit the process extensions from any of the DRG explants. These results demonstrate one of the weaknesses of photolithography, where the process of immobilizing proteins can denature them. However, it is unclear what part of the immobilization process (exposure to UV light or the chemical reaction) had deleterious effects. Another chemical reaction scheme available to immobilize proteins onto biomaterial scaffolds uses UV light to cleave a nitrobenzyl ester bond and expose an alkoxyamine that can react with aldehyde groups on proteins in the encompassing solution (DeForest and Anseth, 2011, 2012; DeForest and Tirrell, 2015). This scheme was used to immobilize collagenase to a PEG-based scaffold and the immobilized collagenase activity was quantified to be $24 \%$ of the soluble enzyme. In addition, immobilized mouse anti-6xHis monoclonal antibody attached to the PEG scaffold retained sufficient bioactivity to recognize a secondary antibody. Finally, vitronectin was immobilized in the PEG scaffolds and encapsulated human mesenchymal stem cells only differentiated in regions where the protein was attached. These studies demonstrate that proteins can be spatially immobilized onto biomaterial scaffolds, but the process does affect biological activity.

\section{Microscale Design in 3D Space}

While most lithographic processes are used for surface-based patterning and protein immobilization on biomaterial scaffolds, there have been several advances that have allowed for patterning in three dimensions. The design of biocompatible materials that are sensitive to UV light has allowed for encapsulating NCs inside biomaterial scaffolds using stereolithography. PC12 cells encapsulated in methacrylate-modified gelatin hydrogels patterned as a crosshatch survived the gelation process and could differentiate 14 days post encapsulation (Zhu et al., 2016). A similar process was used to encapsulate PC12 cells in an acrylate-modified PEG (PEGDA) hydrogel, where cells could differentiate 3 days post encapsulation (Jhaveri et al., 2006). Combining cytocompatible materials with two-photon lithography has allowed for the design of biomaterials with sophisticated 3D architecture (Maruo et al., 1997). Channels with a denser concentration of PEG were created by first crosslinking a large molecular weight PEGDA to form a bulk hydrogel, then leaching in a small molecular weight PEGDA into the scaffold, and finally exposing specific regions in the bulk hydrogel to a two-photon laser for a second round of crosslinking (Hahn et al., 2006). Fluorescent BSA was then leached into the PEG scaffold to demonstrate that regions exposed to the two-photon laser did not allow for BSA diffusion because of the denser network created by crosslinking the small molecular weight PEGDA. The same technique was used to add adhesive domains to the scaffold in various 3D spatial patterns by attaching the RGD polypeptide to the small molecular weight PEGDA before leaching it into the scaffold (Hahn et al., 2006). Two-photon lithography was also used to create a PEG-based scaffold patterned with tunnels inside the scaffold (Livnat et al., 2007). Encapsulated DRG preferentially migrated through the tunnels over 4 days. Two-photon lithography has been combined with other chemical processes to spatially orient bioactive motifs. BSA was immobilized into an HA-based hydrogel in the shape of a spiral using twophoton lithography (Seidlits et al., 2009). The adhesive IKVAV polypeptide was then linked to the immobilized BSA using an avidin-biotin complex. DRG encapsulated in the scaffold and near the immobilized IKVAV polypeptide extended processes toward the IKVAV protein and followed designed spiral pattern. Proteins have been immobilized onto biomaterial scaffolds with a $3 \mathrm{D}$ resolution of a couple of microns, which allows for influencing single cell behavior. BSA was immobilized to a PEGbased scaffold with a resolution of $1 \mu \mathrm{m} \times 1 \mu \mathrm{m}$ in the $x y$-plane and 3-5 $\mu \mathrm{m}$ down the $z$-axis (DeForest and Tirrell, 2015). A similar resolution of $0.5 \mu \mathrm{m}$ radially and $1-2 \mu \mathrm{m}$ down the $z$-axis was achieved for the immobilization of the IKVAV polypeptide (Seidlits et al., 2009). Biomaterial scaffold architecture can be designed at the microscale level by utilizing a combination of lithographic techniques with cytocompatible materials. 


\section{ELECTROSPINNING}

\section{Electrospinning Techniques}

The electrospinning process involves pumping a polymer solution through a charged needle (Pham et al., 2006; Kishan and Cosgriff-Hernandez, 2017). Enough voltage is applied to the needle tip to cause droplets of the polymer solution to overcome the surface tension and emerge from the needle tip in the form of a liquid jet. The liquid jet then undergoes bending instability and rapidly whips into multiple expanding loops. During this whipping process the polymer solution stretches and thins into micrometer and nanometer fibers, and solvents evaporate. The jet is ultimately collected on a grounded or oppositely charged plate that is a variable distance away from the needle tip. The distance of the collector from the needle tip is selected to allow enough time for solvents to evaporate fully and to control the diameter of the fibers. There are several parameters that require careful tuning in order to electrospin a polymer solution into fibers: polymer solution viscosity, conductivity, and flow rate, applied voltage to the needle, and the temperature and humidity of the environment.

Electrospun fibers are patterned by changing the collector used to gather the polymer (Figure 2). A flat plate collector produces fibers with a random orientation. A rotating mandrel is used to align fibers in a singular direction (Matthews et al., 2002). Increasing the speed of the mandrel improves fiber alignment but also affects the mechanical properties of the bulk scaffold formed. Another way to align fibers is to collect them using two conducting electrodes separated by an insulating gap (Li et al., 2004). The gap causes the electrostatic forces to become directional and the fibers align in-between the two electrodes. This method of fiber alignment is limited to thin fiber scaffolds because the fibers carry a charge. As more fiber layers are added the collector starts to behave like a flat plate and subsequent fiber layers become randomly oriented. There are workarounds to this limitation that involve gathering the fibers layer-by-layer and combining them into a single-scaffold post electrospinning (Orr et al., 2015). Electrospun fibers can also be aligned by using two parallel magnets as a collector (Yang et al., 2007). This method is not limited to thin layered scaffolds but does require magnetizing the polymer solution. More sophisticated patterns can be created by adjusting the topography of the collector. Researchers have gathered polyurethane-based fibers on a PDMS collector with a square grid pattern etched onto the surface (Dempsey et al., 2010). The resulting scaffold had aligned fibers along the grid lines and random fibers in the square sections.

\section{NCs on Aligned Fibers}

The alignment of electrospun fibers has a robust effect on NC behavior. Researchers seeded human neural progenitor-derived astrocytes (hNP-AC), human astrocytoma cell line U373, and human neuroblastoma cell line SH-SY5Y on aligned polycaprolactone (PCL) and PCL/collagen-blended fibers (Gerardo-Nava et al., 2009). All three cell types had elongated cell bodies in alignment with fiber direction, extended processes in that direction, and migrated preferentially in parallel with the aligned.
These findings were consistent on both PCL and PCL/collagenblended fiber scaffolds. Human umbilical mesenchymal stem cells (hUMSCs) displayed similar behavior when seeded onto aligned fibrin fibers by orienting actin filaments with the fiber orientation (Figure 4E) (Yao et al., 2016). When hUMSCs were seeded on randomly aligned fibrin fibers they expressed actin filaments in a random orientation, but their morphology did differ from cells cultured on tissue culture plastic controls (Figures 4C,D) (Yao et al., 2016). Alignment of electrospun fibers influences both the direction of NC process extensions and promotes longer processes. DRG seeded on randomly oriented polypyrrole tube fibers extended neurites radially (Xie et al., 2009). By comparison, DRG seeded on aligned polypyrrole tube fibers extended neurites in the directions of the fibers, and displayed a longer maximum neurite length. The effects of aligned fibers are robust and can be repeated across many NC lines.

\section{Fiber Material Composition}

Most biocompatible materials are difficult to electrospin into fibrous scaffolds and some cell lines do not adhere to commonly electrospun polymers like PCL. As a result, many electrospun fiber scaffolds used to seed NCs are either blended with native ECM proteins and polymers or coated with a bioactive material. The effects of material composition on NC behavior are cell line dependent. The adhesion and migration rate of hNP-AC was improved on the PCL/collagen-blended fibers when compared to the PCL fibers (Gerardo-Nava et al., 2009). In contrast, U373 cells did not display a difference in migration rate or adhesion behavior when seeded on PCL/collagen-blended and PCL fiber scaffolds. SH-SY5Y neuroblastoma cells had an increased metabolic activity when seeded on PCL/hyaluronan-blended fibers over PCL fibers (Entekhabi et al., 2016). Electrospun fibers made from PCL blended with gelatin increased C17.2 NSC neurite length over PCL fiber scaffold controls (Ghasemi-Mobarakeh et al., 2008). Fibers made from conductive biomaterials, such as polypyrrole, have gained interest because electrical stimulation also promotes process extensions. Applying an electrical stimulation to randomly oriented and aligned polypyrrole nanotubes increased the DRG maximum neurite length over unstimulated controls (Xie et al., 2009). Electrical stimulation can also increase the number of processes NSCs extend. The percentage of PC12 cells seeded on silk fibroin fibers coated with reduced graphene oxide that extended neurites increased after applying electrical stimulation (Aznar-Cervantes et al., 2017). The increase was comparable to the increase in number of cells with neurites observed when adding soluble nerve growth factor to the media. In addition, multiple rounds of electrical stimulation did not further promote more PC12 cells to extend neurites. NC behavior can also be directed by controlling the electrospun fiber diameter.

\section{Fiber Diameter}

The distance between the charged needle tip and the fiber collector can be adjusted to control fiber diameter. Several 2D studies have investigated the impact of fiber diameter on $\mathrm{NC}$ behavior. Hippocampus-derived adult rat NSCs were seeded on laminin-coated poly(ethersulfone) fibers with 0.283-, 0.749-, 

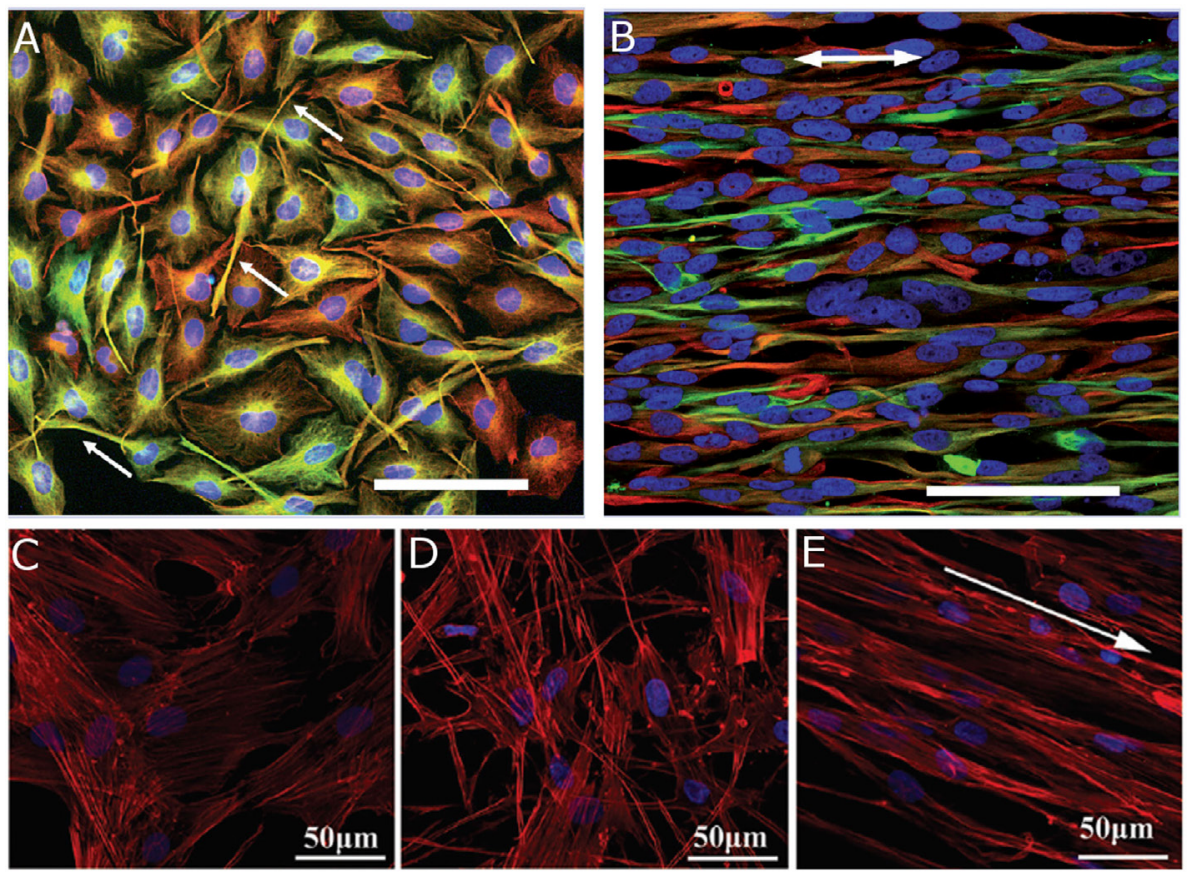

FIGURE 4 | Human astrocytoma cell line U373 cultured on (A) poly-L-lysine substrate for 4 days and (B) aligned PCL fibers for 7 days. Arrows indicate (A) process extensions and (B) fiber orientation. Reproduced with permission of Gerardo-Nava et al. (2009), Copyright 2009, Future Medicine Ltd. Human umbilical cord mesenchymal stem cells cultured on (C) tissue culture plastic, (D) randomly oriented fibrin fibers, and (E) aligned fibrin fibers for 1 day. Arrow indicates fiber orientation. Reproduced with permission of Yao et al. (2016), Copyright 2016, Royal Society of Chemistry. (A,B) Cell nuclei (blue), GFAP (green), vimentin (red), and scale bars $=100 \mu \mathrm{m}$. (C-E) Cell nuclei (blue) and F-actin (red).

and 1.452- $\mu \mathrm{m}$ diameters (Christopherson et al., 2009). In proliferation media conditions, an increase in fiber diameter led to reduced NSC migration, spreading, and proliferation. Under differentiation media conditions, NSCs on $0.283-\mu \mathrm{m}$ diameter fibers preferentially differentiated into oligodendrocytes, while NSCs on 0.749 - and 1.452- $\mu \mathrm{m}$ diameter fibers preferentially differentiated down a neuronal lineage. Differences in electrospun fiber diameter have also been utilized to elucidate oligodendrocyte myelination behavior. Researchers seeded oligodendrocyte precursor cells on poly(L-lysine)-coated polystyrene fibers with diameter ranges of $0.2-0.4$ and $2-4 \mu \mathrm{m}$ (Lee et al., 2012). In part, fibers served as artificial axons, and a higher percentage of the cells cultured on the larger diameter fibers wrapped myelin around the fibers than cells cultured on the smaller diameter fibers. This suggests that axon diameter is an influencing factor in determining which axons are myelinated by oligodendrocytes and which axons are not in the CNS. While electrospinning has been successfully utilized to direct NC behavior on biomaterial scaffolds in 2D culture systems, it has been difficult to translate those results to three dimensions.

\section{Fibers in 3D Scaffolds}

The effects of electrospun fibers have also been investigated in 3D biomaterial scaffolds. Fibers produced by the electrospinning process do not fully mimic the $3 \mathrm{D}$ nature of native tissue. Electrospun fibers are too densely packed to allow for cell infiltration into the biomaterial scaffold. The effects of fiber topography, material composition, and orientation on NCs are limited to surface effects. However, researchers have developed several techniques to incorporate electrospun fibers in $3 \mathrm{D}$ biomaterial scaffolds. A simple way to do this is to gel a hydrogel scaffold on top of the fibers. Researchers gelled a Puramatrixbased hydrogel on top of aligned PCL fibers and investigated if human pluripotent stem cell-derived neuron (hPSC) could sense the presence of the fibers (Hyysalo et al., 2017). hPSCs seeded on top of a 15- $\mu \mathrm{m}$ thick Puramatrix hydrogel could not sense the aligned PCL fibers at the bottom and extended processes with random orientation. hPSCs encapsulated inside the Puramatrix hydrogel and within $10 \mu \mathrm{m}$ of the fibers did align processes along the PCL fiber axis. Cells further away from the PCL fibers, yet still inside the gel, extended processes with a random orientation. A similar study was conducted using stereolithography to gel a square grid patterned PEGDA hydrogel on top of aligned PCL and PCL/gelatin fibers (Lee et al., 2017). Primary cortical neurons and NSCs seeded on top the PEGDA hydrogel did orient process extensions with PCL/gelatin fiber alignment. Although there was no mention of how thick the PEGDA hydrogel was, it suggests that NCs do not need to be in direct contact with aligned fibers to direct behavior. Similar results were obtained by placing PCL, PCL/gelatin, and laminin-coated PCL aligned fibers in the center of HA-based hydrogels using soft lithography (McMurtrey, 2014). Encapsulated SH-5Y5Y neuronal cells grew 
into large spherical clusters away from the fiber layer and had an elongated phenotype at the fiber layer. NCs at the fiber layer extended processes in the direction of the fiber orientation. The average neurite length was quantified and shown to be highest in HA hydrogels with laminin-coated PCL fibers, indicating that cell adhesion to fibers plays a critical role in their behavior. In order get a more uniform cell response across the $z$-axis of 3D biomaterial scaffolds, researchers have stacked multiple fiber layers in-between hydrogel layers. A single-scaffold layer consisted of aligned poly-L,D-lactic acid fibers placed on top of a thin collagen gel with cells seeded on top of the fibers (Weightman et al., 2014). Astrocytes, oligodendrocytes, and oligodendrocyte precursor cells had elongated cell bodies in alignment with fiber direction and extended processes in that direction. It is unclear if this stacking method truly represents a $3 \mathrm{D}$ culture system because cells cannot migrate through the individual fiber layers. The effects of electrospun fibers on directing NSC behavior have been consistent across the in vitro literature, and researchers have begun incorporating them into animal studies.

\section{Fiber Scaffolds In Vivo}

The implementation of electrospun fiber-based biomaterial scaffolds into animal models has not yielded similar results to in vitro experiments. Poly( $\varepsilon$-caprolactone-co-ethyl ethylene phosphate)aligned fibers were incorporated into a collagen hydrogel and implanted into a C5 rat SC incision injury (Milbreta et al., 2016). Endogenous cell response to a collagen scaffold without fibers, a collagen scaffold with fibers in parallel with the SC longitudinal axis, and a collagen scaffold with fibers at an angle to the SC longitudinal axis was analyzed. Collagen scaffolds with fibers had more neurite infiltration and longer neurites 12 weeks post implantation when compared to the collagen scaffold without fibers. However, aligning the fibers in parallel to the SC longitudinal axis did not further improve endogenous cell response. Similar results were found with the implantation of aligned and randomly oriented fibrin fibers into a 4-mm gap thoracic (T9T10) SC hemisection injury (Yao et al., 2016). Endogenous cell infiltration into the aligned fibers did follow the fiber orientation 4 weeks post implant, but total cell infiltration was similar into both randomly oriented and aligned fibrin scaffolds. From these animal studies, it is unclear as to whether or not fiber alignment is important in vivo. Researchers have also investigated the effects of fiber surface charge on endogenous cell infiltration. Polyurethane fibers were plasma coated with films of positive, negative and neural relative surface charge and implanted into rat dorsum for 5 weeks (Sanders et al., 2005). Cell infiltrations into the implants were similar for all conditions, and the study did not investigate infiltrating cell morphology. NCs have also been incorporated with electropun fiber-based scaffolds in vivo. Dopaminergic neurons encapsulated in a xyloglucan hydrogel with short poly(L-lactic acid) fibers were injected into the ventral midbrain of Parkinsonian mice (Wang et al., 2016). There was no difference in transplanted cell viability between xyluglucan scaffolds with and without fibers. Taken together, these findings indicate that the incorporation of scaffolds made from electrospun fibers into animal models have not confirmed in vitro findings and more investigation is necessary.

\section{D BIOPRINTING}

\section{Bioprinting Techniques}

Three-dimensional bioprinting is defined as the layer-by-layer positioning of biomaterials, biochemicals, and cells with spatial control to build a bulk 3D structure. Computer-aided design software is used to control the placement of materials in a syringe, or print head, onto a substrate in the $x$-, $y$-, and $z$-directions. $3 \mathrm{D}$ bioprinting techniques have the potential to mimic the complex micro-architecture of tissue because the biomaterial scaffolds are built using an additive approach and multiple print heads with different biomaterials can be combined to create a single construct (Figure 5). The vast majority of printed biomaterial scaffolds are patterned using the inkjet and microextrusion printing techniques (Murphy and Atala, 2014; Johnson et al., 2016; Ratheesh et al., 2017). Inkjet bioprinting is used to print controlled volumes and works best when printing low-viscosity materials or cells. There are two types of inkjet 3D printing heads, thermal and piezoelectric actuated, that provide similar benefits. Thermal inkjet printing is done by electrically heating the print head to produce a pulse of pressure. Although the localized heat generates temperatures in the range of $200-300^{\circ} \mathrm{C}$, the short time frame of heating $(\sim 2 \mu \mathrm{s})$ only results in a material temperature increase of $4-10^{\circ} \mathrm{C}$ (Cui et al., 2010). While cells can survive the thermal stress, the technique may be incompatible with biomaterials, such as agarose, that undergo a thermal transition to gel or solidify. Piezoelectric actuator inkjet printing is done by applying a voltage to change the actuator shape and produce a droplet of controlled volume. This printing technique does not alter the printed material properties but the mechanical stress produced by the actuator change in shape can induce some cell membrane damage (Chang et al., 2008). Microextrusion 3D printing is used for higher viscosity biomaterials or high cell density applications because inkjet printers are prone to clogging under those conditions. Instead of printing controlled volumes, microextrusion 3D printing applies a force to break the material up into beads in the print head. The force is applied either pneumatically or mechanically with a piston or screw assembly. While there are advantages and disadvantages to each print head set up, they are not limiting for printing biomaterial scaffolds. Microextrusion printers do not
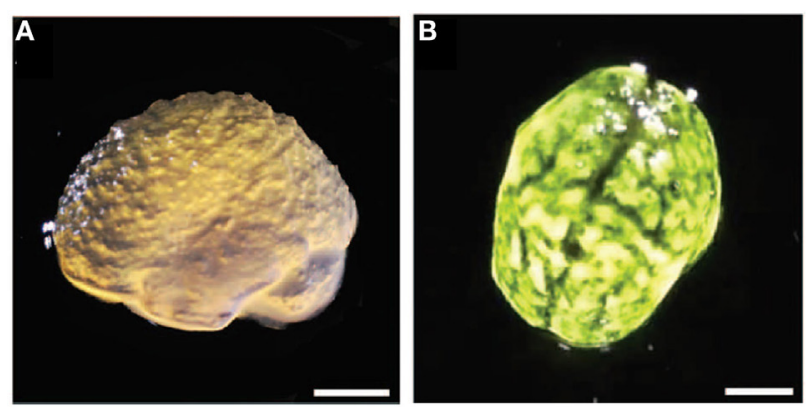

FIGURE 5 | (A) Lateral view of 3D printed alginate gel matching anatomical features of the cortex and cerebellum with microscale resolution. (B) Top view with black dye dripped on alginate gels. Scale bars $=1 \mathrm{~cm}$. Reproduced with permission of Hinton et al. (2015), Creative Commons Copyright (CC-BY). 
have the resolution of inkjet printers and cell viability is lower in some cases (Smith et al., 2004; Cui et al., 2012), but the technique is usable with a larger variety of biomaterials. Ultimately, the print head is selected based on the biomaterial physical properties because scaffold resolution is currently limited by how well biomaterials maintain the printed structure and not the print head capabilities.

For biomaterial applications, the $3 \mathrm{D}$ printing process works best with materials that exhibit yield stress and shear thinning rheological properties (Truby and Lewis, 2016). Materials going through the printing process undergo three stress profiles: low stress while the material is inside the syringe, high stress when the material flows through the print head, and low stress when the printed material is on the stage. Yield-stress materials exhibit two types of responses to stress: when a small stress is applied they deform elastically, maintaining their structure, and when a high stress is applied they deform plastically, causing the material to flow. The transition between the two responses, called the yield point, is material dependent. Any viscous material can be pushed through a print head, but only materials with yield-stress rheological properties will maintain the printed structure on the stage (Malek et al., 2017; Siqueira et al., 2017). Most yield-stress materials also exhibit shear thinning behavior, which helps shield cells from the high shear forces caused by traveling through the print head (Thakur et al., 2016).

\section{Strategies for Biomaterial Printing}

Bioinks are materials that can be $3 \mathrm{D}$ printed, maintain their structure, and are cytocompatible. Most biomaterials do not exhibit favorable rheological properties for $3 \mathrm{D}$ printing so bioinks are developed by simple mixing of existing materials that individually exhibit desired properties, or through chemical modification of existing biomaterials (Lee et al., 2009; Shim et al., 2011; Diamantides et al., 2017). Cortical human NSCs were encapsulated in 3D printed blend of alginate, carboxymethylchitosan, and agarose ( $\mathrm{Gu}$ et al., 2016). The bioink maintained the printed crosshatch structure initially through the cooling of agarose and was subsequently soaked in a calcium chloride solution to crosslink the alginate component. Encapsulated NSCs had an initial cell viability of $\sim 75 \%$ and were able to differentiate into neurons and glial cells. Similar results were found with a bioink of sodium alginate, fibrinogen, and gelatin (Dai et al., 2016). The bioink was soaked in calcium chloride and thrombin solutions after printing to crosslink the sodium alginate and fibrinogen components, respectively. Glioma stem cells encapsulated in the bioink had an initial cell viability of $\sim 85 \%$ and could differentiate into glial and NCs. Cells encapsulated in the printed bioink had a higher resistance to the cancer drug temozolomide than two dimensional controls, highlighting that cells in native $3 \mathrm{D}$ tissue do not behave similar to cell cultures on surfaces. This study also demonstrated the potential of $3 \mathrm{D}$ printing to form hierarchical structures because the crosshatch structure at the microscale level was used to form a bulk scaffold in the shape of a tube. Materials can also be mixed during the printing process. Alginate, matrigel, and human NSCs were printed through a coaxial syringe with three chambers (Alessandri et al., 2016). Human NSCs and matrigel were surrounded by alginate to form capsules and were printed into a calcium chloride bath for crosslinking. Cells extended neurites within the capsules indicating that they were still functional. Ideally, this system would not require alginate to hold the capsule structure of the NSCs in matrigel, but matrigel does not have favorable physical properties for $3 \mathrm{D}$ printing. In addition, the chemical structure of matrigel is not well defined so it would be difficult to chemically modify the structure reliably for 3D printing applications. Biomaterials with well-defined chemical structures do not have this limitation and have been chemically modified to alter their rheological properties for $3 \mathrm{D}$ printing.

Most biomaterials do not exhibit favorable rheological properties for 3D printing so researchers have transformed the rheological properties of biomaterials by functionalizing them with moieties that form physical crosslinks (Lu et al., 2012a; Shepherd et al., 2012; Ouyang et al., 2016). An example of this involves supramolecular bonding of adamantane and $\beta$-cyclodextrin moieties, which assemble into a complex at low stress and disassemble when exposed to high stress (Ouyang et al., 2016). HA scaffolds were formed using supramolecular bonds by modifying the HA macromer with adamantine and $\beta$-cyclodextrin separately, and mixing of the two modified HA solutions together to form the scaffold (Ouyang et al., 2016). Modified HA was successfully printed into a crosshatch pattern, but the scaffold could only maintain the crosshatch structure when few layers were printed. In order to prevent the scaffold from collapsing from the stress caused by overlaying layers and maintain the crosshatch pattern at higher printing heights, HA was further modified with methacrylate groups. HA hydrogels crosslinked with both the supramolecular assembly and chemical methacrylate bonds, after being exposed to UV light, maintained their printed structure for up to one month with as many as 16 printed layers. This study also highlights the importance of gelation kinetics for maintaining printed structure integrity. Only the scaffolds that were exposed to additional UV light in the presence of a photoinitiator after the printing process was complete maintained their structure for 1 month. This indicated that UV exposure during the printing process did not fully crosslink methacrylate groups. Crosslinking kinetics are important considerations for 3D printing biomaterials.

Researchers have investigated inducing fast gelation kinetics in biomaterials to avoid requiring yield-stress rheological properties to maintain the printed structure. Gellan gum was printed using a coaxial syringe needle set up, with the gellan gum solution in the inner syringe ring and a crosslinking solution on the outer ring (Lozano et al., 2015). The material was not printed into a pattern, instead layers with and without encapsulated primary cortical neurons were alternately printed on top of each other. Encapsulated primary cortical neurons had a viability of $70-80 \%$, differentiated into neurons and glia, and extended processes into up to $100 \mu \mathrm{m}$ into layers without cells 5 days after printing. Waterborne polyurethane, which undergoes gelation through a temperature transition at $37^{\circ} \mathrm{C}$, was successfully $3 \mathrm{D}$ printed by mixing with soy protein isolate to reduce the gelation time and printing onto a heated stage (Hsieh et al., 2015; Lin et al., 2016). Up to eight layers were printed into a crosshatch pattern, and encapsulated murine NSCs had high viability 3 days after printing. However, cell metabolic activity was lower than tissue 
culture polystyrene controls, indicating that the printing process had a negative impact on the murine NSCs.

\section{Bioprinting Bioactive Molecules}

The $3 \mathrm{D}$ bioprinting technique is not limited to printing bioinks with cells, proteins, and other bioactive molecules can also be incorporated. Growth factors incorporated with bioinks maintain protein bioactivity after being printed. Fibroblast growth factor- 2 (FGF2) and ciliary neurotrophic factor (CNTF) were printed onto polyacrylamide hydrogels, and primary fetal NSCs were subsequently seeded onto the hydrogels to demonstrate that the growth factors remained biologically active after printing (Ilkhanizadeh et al., 2007). Soluble FGF2 promotes proliferation and NSCs seeded on portions of the gel with FGF2 did not differentiate into glial cells. Soluble CNTF promotes differentiation and NSCs did differentiate into glial cells when seeded on portions of the hydrogel with CNTF. The amount of NSC differentiation into glial cells could be spatially controlled by printing a concentration gradient of CNTF across the polyacrylamide scaffold. 3D bioprinting has the potential to be utilized as a local drug delivery mechanism because the printed pattern affects cumulative drug release. The chemotherapeutic drug 5-fluorouracil was printed with a bioink composed of PLGA and PCL (Yi et al., 2016). The cumulative release of the drug from the printed scaffold depended on both the printed pore structure and the number of printed layers in the $z$-direction. Combinations of bioinks with growth factors have directed NSC behavior. A fibrin hydrogel was printed with vascular endothelial growth factor (VEGF) and placed next to a collagen gel with murine NSCs encapsulated (Lee et al., 2010). The NSCs migrated and proliferated toward the fibrin hydrogel when VEGF was incorporated and did not do so when fibrin was printed without VEGF.

\section{FUTURE PERSPECTIVES}

Advances in microscale patterning of biomaterial scaffolds have allowed researchers to investigate NC behavior in response to both physical and biochemical environmental cues. Topographical cues such as grooves and aligned fibers can direct NSC differentiation and neural process extensions. Similar effects can also be biochemically induced by spatially patterning adhesive motifs. Microscale patterning of biomaterial scaffolds has been performed predominantly by the use of lithography, electrospinning, and $3 \mathrm{D}$ bioprinting techniques. The final goal remains to develop microarchitecture to direct NC behavior for in vivo therapeutic treatments and tissue regeneration, and the immediate challenges for further developing each technique vary significantly.

Lithography has been successfully implemented to pattern grooves on surfaces, channels within biomaterial scaffolds, and spatially immobilize proteins to scaffolds. It has proved to be a powerful tool for developing in vitro models to mimic some aspects of native tissue at the microscale level. However, it will be challenging to translate the techniques developed for in vitro models to in vivo applications. The use of light as a source for patterning at the microscale level is inherently limited to thin or transparent scaffolds. Optically transparent materials, such as PEG, can be patterned well using light but scaffolds made from proteins, such as collagen, are optically dense and cannot be patterned deep within the scaffold. This limitation will be significant when attempting to translate these scaffolds to large animal studies and clinical trials because the biomaterial scaffolds will need to be scaled to appropriate sizes. In addition, current protein immobilization techniques require incubating the biomaterial scaffold in the protein of interest and then using a light source to initiate the immobilization reaction. This has limited application in vivo because it takes hours for large proteins, such as growth factors, to diffuse into the scaffold and the process requires several rinse steps. For this technique to be used in a transplant in vivo the scaffold must be designed in vitro and then transplanted. While applicable for acellular "device" implants, this method has several drawbacks when incorporating NC into the scaffolds. For example, encapsulated NCs may uptake the protein during the incubation step and not allow for the protein to be dispersed throughout the scaffold as desired for immobilization. These issues do not limit investigating the effects of microscale architecture on NC behavior in in vitro models but do hamper translation into in vivo studies.

Electrospinning of fibers into an aligned orientation affects a wide range of cell lines in a similar fashion in a 2D setting. NCs elongate cell bodies, extend processes, and migrate preferentially in alignment with fiber scaffold orientation. However, electrospun fibers have not yet been translated into homogeneous 3D biomaterial scaffolds, and differences in NC behavior have been observed when moving from $2 \mathrm{D}$ culture systems to 3D culture systems (Lampe et al., 2010; Bozza et al., 2014; Park et al., 2014; Shin et al., 2014). Current attempts at creating 3D biomaterial scaffolds from electrospun fiber are limited to layering a fiber mesh sandwiched between gels or in-between another biomaterial and NCs. While the bulk material is 3D, this method does not allow for cells to readily embed past a single layer in the $z$-direction because electrospun fiber meshes are generally too dense for deep cell infiltration. Cell infiltration into the fiber mesh can be improved by electrospinning sacrificial fibers alongside the main fiber material (Baker et al., 2008). The sacrificial fibers are dissolved in solution after electrospinning, leaving behind a more porous fiber mesh. Attempts at incorporating electrospun fibers into animal studies have not induced similar NC behavior as observed in vitro (Sanders et al., 2005; Milbreta et al., 2016; Wang et al., 2016; Yao et al., 2016). The challenge for the field is to recapitulate the desired NC behavior observed on 2D fiber meshes into 3D biomaterial scaffolds. Collector topography has been used to pattern fiber scaffolds into more sophisticated patterns than simply aligning fibers (Dempsey et al., 2010; Kishan et al., 2017), and may become a useful tool in creating a porous $3 \mathrm{D}$ fiber mesh. The alignment of fibers has the potential to direct NC process extensions in vivo, which has many applications like aligning neural synapses along a damaged SC. However, this potential using electrospun materials has yet to be realized in a 3D culture system. The problem can be tackled by either improving the porosity of the electrospun fibers, incorporating cells in the electrospinning process (Stankus et al., 2006; Zanatta et al., 2012), or by combining electrospinning with another technique such as $3 \mathrm{D}$ bioprinting. 
Three-dimensional bioprinting has the most potential for developing tissue-like biomaterial scaffolds because it is an additive process. However, 3D bioprinting of scaffolds is currently limited by the available biomaterials. The printing process requires cytocompatible materials that exhibit yield stress and shear thinning rheological properties. Research has focused on blending biomaterials with materials that exhibit the desired rheological properties, or modifying biomaterials chemically. However, simple blending of materials and chemical modifications have many limitations. For example, the blend ratios have a drastic impact on the rheological properties of bioinks created by simply mixing materials. Adding a new protein or bioactive cue to the mixture may alter the rheological properties of the bioink enough to where it is no longer printable (Shim et al., 2011). Chemical modifications are limited to materials that have a well-defined chemical structure and materials such as collagen are challenging to modify reliably and repeatable. The current challenge is developing biomaterials with favorable properties for $3 \mathrm{D}$ printing. A universal way to tackle these issues is to use a sacrificial material to act as a mold to contain the biomaterial scaffold until it is solidified through crosslinking (Hinton et al., 2015). Using sacrificial materials allows for $3 \mathrm{D}$ printing a plethora of biomaterials with differing crosslinking kinetics but also slows down the printing process since the sacrificial material needs to be printed in conjunction to the desired biomaterial. The development of suitable biomaterials for 3D bioprinting will remain a challenge in the field for the foreseeable future.

A major challenge in tissue engineering is to develop microscale architectures that will lead to therapeutic treatments of CNS injury and disease. Microscale architecture can be used to spatially direct NC differentiation, process extensions, and migration. However, the field is uncertain as to how $\mathrm{NC}$ behavior should be directed and which NC line should be used for therapeutic treatments. For example, for repair of SC contusion injury it is important to replace damaged neurons, but that is not the only cell type required to restore functionality. When designing a biomaterial scaffold, should NSC be directed solely toward a neuronal pathway or are other cell types, such as oligodendrocytes

\section{REFERENCES}

Alessandri, K., Feyeux, M., Gurchenkov, B., Delgado, C., Trushko, A., Krause, K.-H., et al. (2016). A 3D printed microfluidic device for production of functionalized hydrogel microcapsules for culture and differentiation of human neuronal stem cells (hNSC). Lab. Chip 16, 1593-1604. doi:10.1039/C6LC00133E

Arvidsson, A., Collin, T., Kirik, D., Kokala, Z., and Lindvall, O. (2002). Neuronal replacement from endogenous precursors in the adult brain after stroke. Nat. Med. 8, 963-970. doi:10.1038/nm

Aznar-Cervantes, S., Pagán, A., Martínez, J. G., Bernabeu-Esclapez, A., Otero, T. F., Meseguer-Olmo, L., et al. (2017). Electrospun silk fibroin scaffolds coated with reduced graphene promote neurite outgrowth of PC-12 cells under electrical stimulation. Mater. Sci. Eng. C 79, 315-325. doi:10.1016/j.msec.2017.05.055

Baker, B. M., Gee, A. O., Metter, R. B., Nathan, A. S., Marklein, R. A., Burdick, J. A., et al. (2008). The potential to improve cell infiltration in composite fiber-aligned electrospun scaffolds by the selective removal of sacrificial fibers. Biomaterials 29, 2348-2358. doi:10.1016/j.biomaterials.2008.01.032

Bakshi, A., Keck, C. A., Koshkin, V. S., Lebold, D. G., Siman, R., Snyder, E. Y., et al. (2005). Caspase-mediated cell death predominates following engraftment of neural progenitor cells into traumatically injured rat brain. Brain Res. 1065, 8-19. doi:10.1016/j.brainres.2005.09.059 and/or astrocytes, also required? In addition, are NSC the best cell type to transplant or would a scaffold with several encapsulated NPC lines be more effective? Encapsulating NCs in biomaterial scaffolds with microscale architecture in an in vitro setting may help answer some of these questions. For example, in order to determine how NSC differentiation should be directed for transplant therapies it is important to investigate how each cell line remodels the ECM. However, the CNS ECM is complex, difficult to isolate intact and challenging to characterize. By immobilizing individual proteins onto a scaffold NC behavior can be investigated on an individual protein basis. Spatial control of NCs and proteins within biomaterial scaffolds will help create reductionist in vitro models of in vivo features such as the glial scar which may help elucidate the importance of specific interactions. A common challenge shared by research using all three techniques discussed here is the development of relevant microscale patterns. For example, can astrocytes, neurons and proteins be organized at the microscale level to mimic glial scar behavior in vitro? Currently there are few patterns that accomplish this because most research has focused on developing the capability to control microscale architecture and refining the resolution. Finding a clear link between microscale patterning techniques and physiological relevance remains a challenge for the neural tissue engineering field to meet. As the technical challenges of patterning biomaterial scaffolds at the microscale level are now being overcome, meaningful designs and patterns must be achieved that replicate the complicated architecture of native tissue in order to improve NC treatment therapies.

\section{AUTHOR CONTRIBUTIONS}

EM and KL wrote and edited the manuscript.

\section{FUNDING}

This work was supported by the University of Virginia, and the School of Engineering and Applied Science Research Innovation Award.

Barnabé-Heider, F., Göritz, C., Sabelström, H., Takebayashi, H., Pfrieger, F. W., Meletis, K., et al. (2010). Origin of new glial cells in intact and injured adult spinal cord. Cell Stem Cell 7, 470-482. doi:10.1016/j.stem.2010.07.014

Béduer, A., Vieu, C., Arnauduc, F., Sol, J. C., Loubinoux, I., and Vaysse, L. (2012). Engineering of adult human neural stem cells differentiation through surface micropatterning. Biomaterials 33, 504-514. doi:10.1016/j. biomaterials.2011.09.073

Bozza, A., Coates, E. E., Incitti, T., Ferlin, K. M., Messina, A., Menna, E., et al. (2014). Neural differentiation of pluripotent cells in 3D alginate-based cultures. Biomaterials 35, 4636-4645. doi:10.1016/j.biomaterials.2014.02.039

Chang, R., Nam, J., and Sun, W. (2008). Effects of dispensing pressure and nozzle diameter on cell survival from solid freeform fabrication-based direct cell writing. Tissue Eng. Part A 14, 41-48. doi:10.1089/ten.a.2007.0004

Chedly, J., Soares, S., Montembault, A., von Boxberg, Y., Veron-Ravaille, M., Mouffle, C., et al. (2017). Physical chitosan microhydrogels as scaffolds for spinal cord injury restoration and axon regeneration. Biomaterials 138, 91-107. doi:10.1016/j.biomaterials.2017.05.024

Christopherson, G. T., Song, H., and Mao, H. Q. (2009). The influence of fiber diameter of electrospun substrates on neural stem cell differentiation and proliferation. Biomaterials 30, 556-564. doi:10.1016/j.biomaterials. 2008.10.004 
Ciuciu, A. I., and Cywiński, P. J. (2014). Two-photon polymerization of hydrogels-versatile solutions to fabricate well-defined 3D structures. RSC Adv. 4, 45504-45516. doi:10.1039/c4ra06892k

Cooke, M. J., Vulic, K., and Shoichet, M. S. (2010). Design of biomaterials to enhance stem cell survival when transplanted into the damaged central nervous system. Soft Matter 6, 4988. doi:10.1039/c0sm00448k

Correale, J., Farez, M. F., and Cardona, A. E. (2015). The role of astrocytes in multiple sclerosis progression. Front. Neurol. 6:1-12. doi:10.3389/fneur.2015.00180

Cui, X., Boland, T., D'Lima, D. D., and Lotz, M. K. (2012). Thermal inkjet printing in tissue engineering and regenerative medicine. Recent Pat. Drug Deliv. Formul. 6, 149-155. doi:10.1086/498510.Parasitic

Cui, X., Dean, D., Ruggeri, Z. M., and Boland, T. (2010). Cell damage evaluation of thermal inkjet printed Chinese hamster ovary cells. Biotechnol. Bioeng. 106, 963-969. doi:10.1002/bit.22762

Curley, J. L., Catig, G. C., Horn-Ranney, E. L., and Moore, M. J. (2014). Sensory axon guidance with semaphorin $6 \mathrm{~A}$ and nerve growth factor in a biomimetic choice point model. Biofabrication 6, 35026. doi:10.1088/1758-5082/6/3/ 035026

Dai, X., Ma, C., Lan, Q., and Xu, T. (2016). 3D bioprinted glioma stem cells for brain tumor model and applications of drug susceptibility. Biofabrication 8, 45005. doi:10.1088/1758-5090/8/4/045005

DeForest, C. A., and Anseth, K. S. (2011). Cytocompatible click-based hydrogels with dynamically tunable properties through orthogonal photoconjugation and photocleavage reactions. Nat. Chem. 3, 925-931. doi:10.1038/nchem.1174

DeForest, C. A., and Anseth, K. S. (2012). Photoreversible patterning of biomolecules within click-based hydrogels. Angew. Chem. Int. Ed. 51, 1816-1819. doi:10.1002/anie.201106463

DeForest, C. A., and Tirrell, D. A. (2015). A photoreversible protein-patterning approach for guiding stem cell fate in three-dimensional gels. Nat. Mater. 14, 523-531. doi:10.1038/nmat4219

Dell'Albani, P. (2008). Stem cell markers in gliomas. Neurochem. Res. 33, 2407-2415. doi:10.1007/s11064-008-9723-8

Dempsey, D. K., Schwartz, C. J., Ward, R. S., Iyer, A. V., Parakka, J. P., and CosgriffHernandez, E. M. (2010). Micropatterning of electrospun polyurethane fibers through control of surface topography. Macromol. Mater. Eng. 295, 990-994. doi:10.1002/mame.201000152

Diamantides, N., Wang, L., Pruiksma, T., Siemiatkoski, J., Dugopolski, C., Shortkroff, S., et al. (2017). Correlating rheological properties and printability of collagen bioinks: the effects of riboflavin photocrosslinking and $\mathrm{pH}$. Biofabrication 9, 34102. doi:10.1088/1758-5090/aa780f

Dos Reis, G., Fenili, F., Gianfelice, A., Bongiorno, G., Marchesi, D., Scopelliti, P. E., et al. (2010). Direct microfabrication of topographical and chemical cues for the guided growth of neural cell networks on polyamidoamine hydrogels. Macromol. Biosci. 10, 842-852. doi:10.1002/mabi.200900410

Dye, B. R., Dedhia, P. H., Miller, A. J., Nagy, M. S., White, E. S., Shea, L. D., et al. (2016). A bioengineered niche promotes in vivo engraftment and maturation of pluripotent stem cell derived human lung organoids. Elife 5, 1-18. doi:10.7554/ eLife. 19732

Entekhabi, E., Haghbin Nazarpak, M., Moztarzadeh, F., and Sadeghi, A. (2016). Design and manufacture of neural tissue engineering scaffolds using hyaluronic acid and polycaprolactone nanofibers with controlled porosity. Mater. Sci. Eng. C 69, 380-387. doi:10.1016/j.msec.2016.06.078

Gerardo-Nava, J., Führmann, T., Klinkhammer, K., Seiler, N., Mey, J., Klee, D., et al. (2009). Human neural cell interactions with orientated electrospun nanofibers in vitro. Nanomedicine 4, 11-30. doi:10.2217/17435889.4.1.11

Ghasemi-Mobarakeh, L., Prabhakaran, M. P., Morshed, M., Nasr-Esfahani, M. H., and Ramakrishna, S. (2008). Electrospun poly( $\varepsilon$-caprolactone)/gelatin nanofibrous scaffolds for nerve tissue engineering. Biomaterials 29, 4532-4539. doi:10.1016/j.biomaterials.2008.08.007

Gordon, J., Amini, S., and White, M. K. (2013). General overview of neuronal cell culture. Methods Mol. Biol. 1078, 1-6. doi:10.1007/978-1-62703-640-5

Gu, Q., Tomaskovic-Crook, E., Lozano, R., Chen, Y., Kapsa, R. M., Zhou, Q., et al. (2016). Functional 3D neural mini-tissues from printed gel-based bioink and human neural stem cells. Adv. Healthc. Mater. 5, 1429-1438. doi:10.1002/ adhm.201600095

Gunther, M. I., Weidner, N., Muller, R., and Blesch, A. (2015). Cell-seeded alginate hydrogel scaffolds promote directed linear axonal regeneration in the injured rat spinal cord. Acta Biomater. 27, 140-150. doi:10.1016/j.actbio.2015.09.001
Hahn, M. S., Miller, J. S., and West, J. L. (2006). Three-dimensional biochemical and biomechanical patterning of hydrogels for guiding cell behavior. $A d v$. Mater. Weinheim 18, 2679-2684. doi:10.1002/adma.200600647

Hahn,M.S., Taite,L.J.,Moon,J. J., Rowland,M.C., Ruffino, K.A., andWest,J. L.(2006). Photolithographic patterning of polyethylene glycol hydrogels. Biomaterials 27, 2519-2524. doi:10.1016/j.biomaterials.2005.11.045

Haring, A. P., Sontheimer, H., and Johnson, B. N. (2017). Microphysiological human brain and neural systems-on-a-chip: potential alternatives to small animal models and emerging platforms for drug discovery and personalized medicine. Stem Cell Rev. Rep. 13, 381-406. doi:10.1007/s12015-017-9738-0

Hill, C. E., Moon, L. D. F., Wood, P. M., and Bunge, M. B. (2006). Labeled Schwann cell transplantation: cell loss, host Schwann cell replacement, and strategies to enhance survival. Glia 53, 338-343. doi:10.1002/glia.20287

Hinton, T. J., Jallerat, Q., Palchesko, R. N., Park, J. H., Grodzicki, M. S., Shue, H.-J., et al. (2015). Three-dimensional printing of complex biological structures by freeform reversible embedding of suspended hydrogels. Sci. Adv. 1, e1500758. doi:10.1126/sciadv.1500758

Horn-ranney, E. L., Curley, J. L., Catig, G. C., Huval, R. M., and Michael, J. (2014). Structural and molecular micropatterning of dual hydrogel constructs for neural growth models using photochemical strategies. Biomed. Microdevices 15, 49-61. doi:10.1007/s10544-012-9687-y

Hsieh, F. Y., Lin, H. H., and Hsu, S. H. (2015). 3D bioprinting of neural stem cell-laden thermoresponsive biodegradable polyurethane hydrogel and potential in central nervous system repair. Biomaterials 71, 48-57. doi:10.1016/j.biomaterials. 2015.08.028

Huang, L., Wu, Z., Zhuge, Q., Zheng, W., Shao, B., Wang, B., et al. (2014). Glial scar formation occurs in the human brain after ischemic stroke. Int. J. Med. Sci. 11, 344-348. doi:10.7150/ijms.8140

Huval, R. M., Miller, O. H., Curley, J. L., Fan, Y., Hall, B. J., and Moore, M. J. (2015). Microengineered peripheral nerve-on-a-chip for preclinical physiological testing. Lab. Chip 15, 2221-2232. doi:10.1039/C4LC01513D

Hynd, M. R., Frampton, J. P., Burnham, M.-R., Martin, D. L., Dowell-mesfin, N., Turner, J. N., et al. (2006). Functionalized hydrogel surfaces for the patterning of multiple biomolecules. J. Biomed. Mater. Res. A 81, 347-354. doi:10.1002/ jbm.a.31002

Hynd, M. R., Frampton, J. P., Dowell-mesfin, N., Turner, J. N., and Shain, W. (2009). Directed cell growth on protein functionalized hydrogel surfaces. J. Neurosci. Methods 162, 255-263. doi:10.1016/j.jneumeth.2007.01.024

Hyysalo, A., Ristola, M., Joki, T., Honkanen, M., Vippola, M., and Narkilahti, S. (2017). Aligned poly( $\varepsilon$-caprolactone) nanofibers guide the orientation and migration of human pluripotent stem cell-derived neurons, astrocytes, and oligodendrocyte precursor cells in vitro. Macromol. Biosci. 17, 1-8. doi:10.1002/ mabi.201600517

Ilkhanizadeh, S., Teixeira, A. I., and Hermanson, O. (2007). Inkjet printing of macromolecules on hydrogels to steer neural stem cell differentiation. Biomaterials 28, 3936-3943. doi:10.1016/j.biomaterials.2007.05.018

Jang, J., Song, Y., Yoo, D., Ober, C. K., Lee, J., and Lee, T. (2016). The development of fluorous photolithographic materials and their applications to achieve flexible organic electronic devices. Flexible Printed Electron. 1, 1-16. doi:10.1088/2058-8585/1/2/023001

Jhaveri, S. J., Senaratne, W., Hynd, M. R., Turner, J. N., Sengupta, P., Shain, W., et al. (2006). Defining the biology-materials interface using both 2D and 3D lithography. J. Photopolym. Sci. Technol. 19, 435-440. doi:10.2494/photopolymer.19.435

Johnson, B. N., Lancaster, K. Z., Hogue, I. B., Meng, F., Kong, Y. L., Enquist, L. W., et al. (2016). 3D printed nervous system on a chip. Lab. Chip 16, 1393-1400. doi:10.1039/C5LC01270H

Kaneko, A., Matsushita, A., and Sankai, Y. (2015). A 3D nanofibrous hydrogel and collagen sponge scaffold promotes locomotor functional recovery, spinal repair, and neuronal regeneration after complete transection of the spinal cord in adult rats. Biomed. Mater. 10, 15008. doi:10.1088/1748-6041/10/1/015008

King, V. R., Henseler, M., Brown, R. A., and Priestley, J. V. (2003). Mats made from fibronectin support oriented growth of axons in the damaged spinal cord of the adult rat. Exp. Neurol. 182, 383-398. doi:10.1016/S0014-4886(03) 00033-5

King, V. R., Phillips, J. B., Hunt-Grubbe, H., Brown, R., and Priestley, J. V. (2006). Characterization of non-neuronal elements within fibronectin mats implanted into the damaged adult rat spinal cord. Biomaterials 27, 485-496. doi:10.1016/j.biomaterials.2005.06.033 
Kishan, A. P., and Cosgriff-Hernandez, E. M. (2017). Recent advancements in electrospinning design for tissue engineering applications: a review. J. Biomed. Mater. Res. A 105, 2892-2905. doi:10.1002/jbm.a.36124

Kishan, A. P., Robbins, A. B., Mohiuddin, S. F., Jiang, M., Moreno, M. R., and Cosgriff-Hernandez, E. M. (2017). Fabrication of macromolecular gradients in aligned fiber scaffolds using a combination of in-line blending and air-gap electrospinning. Acta Biomater. 56, 118-128. doi:10.1016/j.actbio.2016.12.041

Krause, G. S., White, B. C., Aust, S. D., Nayini, N. R., and Kumar, K. (1988). Brian cell death following ischemia and reperfusion: a proposed biochemical sequence. Crit. Care Med. 16, 714-726. doi:10.1097/00003246-198807000-00015

Lampe, K. J., Bjugstad, K. B., and Mahoney, M. J. (2010). Impact of degradable macromer content in a poly (ethylene glycol) hydrogel on neural cell metabolic acitvity, redox state, proliferation, and differentiation. Tissue Eng. Part A 16, 1857-1866. doi:10.1089/ten.tea.2009.0509

Lee, M. K., Rich, M. H., Baek, K., Lee, J., and Kong, H. (2015). Bioinspired tuning of hydrogel permeability-rigidity dependency for 3D cell culture. Sci. Rep. 5, 8948. doi: $10.1038 /$ srep08948

Lee, S., Leach, M. K., Redmond, S. A., Chong, S. Y. C., Mellon, S. H., Tuck, S. J., et al. (2012). A culture system to study oligodendrocyte myelination processes using engineered nanofibers. Nat. Methods 9, 917-922. doi:10.1038/nmeth.2105

Lee, S., Nowicki, M., Harris, B., and Zhang, L. G. (2017). Fabrication of a highly aligned neural scaffold via a table top stereolithography $3 \mathrm{D}$ printing and electrospinning. Tissue Eng. Part A 23, 491-502. doi:10.1089/ten.tea.2016.0353

Lee, W., Pinckney, J., Lee, V., Lee, J., Fischer, K., Polio, S., et al. (2009). Threedimensional bioprinting of rat embryonic neural cells. Neuroreport 20, 798-803. doi:10.1097/WNR.0b013e32832b8be4

Lee, Y. B., Polio, S., Lee, W., Dai, G., Menon, L., Carroll, R. S., et al. (2010). Bioprinting of collagen and VEGF-releasing fibrin gel scaffolds for neural stem cell culture. Exp. Neurol. 223, 645-652. doi:10.1016/j.expneurol.2010.02.014

Li, D., Wang, Y., and Xia, Y. (2004). Electrospinning nanofibers as uniaxially aligned arrays and layer-by-layer stacked films. Adv. Mater. Weinheim 16, 361-366. doi:10.1002/adma.200306226

Lin, H., Hsieh, F., Tseng, C., and Hsu, S. (2016). Preparation and characterization of a biodegradable polyurethane hydrogel and the hybrid gel with soy protein for 3D cell-laden bioprinting. J. Mater. Chem. B 4, 6694-6705. doi:10.1039/ C6TB01501H

Lipinski, B. (2011). Hydroxyl radical and its scavengers in health and disease. Oxid. Med. Cell. Longev. 2011, 8089696. doi:10.1155/2011/809696

Livnat, N., Sarig-Nadir, O., Zajdman, R., Seliktar, D., and Shoham, S. (2007). "A hydrogel-based nerve regeneration conduit with sub-micrometer feature control," in Proceedings of the 3rd International IEEE EMBS Conference on Neural Engineering. Kohala Coast, HI, 101-103.

Lozano, R., Stevens, L., Thompson, B. C., Gilmore, K. J., Gorkin, R., Stewart, E. M., et al. (2015). 3D printing of layered brain-like structures using peptide modified gellan gum substrates. Biomaterials 67, 264-273. doi:10.1016/j. biomaterials.2015.07.022

Lu, H. D., Charati, M. B., Kim, I. L., and Burdick, J. A. (2012a). Injectable shear-thinning hydrogels engineered with a self-assembling dock-and-lock mechanism. Biomaterials 33, 2145-2153. doi:10.1016/j.biomaterials.2011.11.076

Lu, P., Wang, Y., Graham, L., McHale, K., Gao, M., Wu, D., et al. (2012b). Longdistance growth and connectivity of neural stem cells after severe spinal cord injury. Cell 150, 1264-1273. doi:10.1016/j.cell.2012.08.020

Luo, Y., and Shoichet, M. S. (2004a). A photolabile hydrogel for guided three-dimensional cell growth and migration. Nat. Mater. 3, 249-253. doi:10.1038/ nmat1092

Luo, Y., and Shoichet, M. S. (2004b). Light-activated immobilization of biomolecules to agarose hydrogels for controlled cellular response. Biomacromolecules 5, 2315-2323. doi:10.1021/bm0495811

Lynam, D. A., Shahriari, D., Wolf, K. J., Angart, P. A., Koffler, J., Tuszynski, M. H., et al. (2015). Brain derived neurotrophic factor release from layer-bylayer coated agarose nerve guidance scaffolds. Acta Biomater. 18, 128-131. doi:10.1016/j.actbio.2015.02.014

Macaya, D., and Spector, M. (2012). Injectable hydrogel materials for spinal cord regeneration: a review. Biomed. Mater. 7, 12001. doi:10.1088/1748-6041/7/1/ 012001

Malek, S., Raney, J. R., Lewis, J. A., and Gibson, L. J. (2017). Lightweight 3D cellular composites inspired by balsa. Bioinspir. Biomim. 12, 26014. doi:10.1088/1748-3190/aa6028
Maruo, S., Nakamura, O., and Kawata, S. (1997). Three-dimensional microfabrication with two-photon-absorbed photopolymerization. Opt. Lett. 22, 132-134. doi:10.1364/OL.22.000132

Matthews, J. A., Wnek, G.E.,Simpson,D.G., andBowlin,G.L.(2002).Electrospinning of collagen nanofibers. Biomacromolecules 3, 232-238. doi:10.1021/ bm015533u

Mattotti,M., Alvarez,Z., Ortega,J. A.,Planell,J. A., Engel,E., and Alcántara,S.(2012). Inducing functional radial glia-like progenitors from cortical astrocyte cultures using micropatterned PMMA. Biomaterials 33, 1759-1770. doi:10.1016/ j.biomaterials.2011.10.086

McCormick, A. M., Maddipatla, M. V. S. N., Shi, S., Chamsaz, E. A., Yokoyama, H., Joy, A., et al. (2014). Micropatterned coumarin polyester thin films direct neurite orientation. ACS Appl. Mater. Interfaces 6, 19655-19667. doi:10.1021/ am5044328

McMurtrey, R. J. (2014). Patterned and functionalized nanofiber scaffolds in three-dimensional hydrogel constructs enhance neurite outgrowth and directional control. J. Neural Eng. 11, 66009. doi:10.1088/1741-2560/11/6/066009

Milbreta, U., Nguyen, L. H., Diao, H., Lin, J., Wu, W., Sun, C. Y., et al. (2016). Three-dimensional nanofiber hybrid scaffold directs and enhances axonal regeneration after spinal cord injury. ACS Biomater. Sci. Eng. 2, 1319-1329. doi:10.1021/acsbiomaterials.6b00248

Murphy, S. V., and Atala, A. (2014). 3D bioprinting of tissues and organs. Nat. Biotechnol. 32, 773-785. doi:10.1038/nbt.2958

Murry, C. E., and Keller, G. (2008). Differentiation of embryonic stem cells to clinically relevant populations: lessons from embryonic development. Cell 132, 661-680. doi:10.1016/j.cell.2008.02.008

Musoke-Zawedde, P., and Shoichet, M. S. (2006). Anisotropic three-dimensional peptide channels guide neurite outgrowth within a biodegradable hydrogel matrix. Biomed. Mater. 1, 162-169. doi:10.1088/1748-6041/1/3/011

Nagamine, K., Hirata, T., Okamoto, K., Abe, Y., Kaji, H., and Nishizawa, M. (2015). Portable micropatterns of neuronal cells supported by thin hydrogel films. ACS Biomater. Sci. Eng. 1, 329-334. doi:10.1021/acsbiomaterials.5b00020

Nakagomi, T., Taguchi, A., Fujimori, Y., Saino, O., Nakano-Doi, A., Kubo, S., et al. (2009). Isolation and characterization of neural stem/progenitor cells from post-stroke cerebral cortex in mice. Eur. J. Neurosci. 29, 1842-1852. doi:10.1111/j.1460-9568.2009.06732.x

Oh, J., Recknor, J. B., Recknor, J. C., Mallapragada, S. K., and Sakaguchi, D. S. (2009). Soluble factors from neocortical astrocytes enhance neuronal differentiation of neural progenitor cells from adult rat hippocampus on micropatterned polymer substrates. J. Biomed. Mater. Res. A 91, 575-585. doi:10.1002/jbm.a.32242

Orr, S. B., Chainani, A., Hippensteel, K. J., Kishan, A., Gilchrist, C., Garrigues, N. W., et al. (2015). Aligned multilayered electrospun scaffolds for rotator cuff tendon tissue engineering. Acta Biomater. 24, 117-126. doi:10.1016/j.actbio.2015.06.010

Ouyang, L., Highley, C. B., Rodell, C. B., Sun, W., and Burdick, J. A. (2016). 3D printing of shear-thinning hyaluronic acid hydrogels with secondary cross-linking. ACS Biomater. Sci. Eng. 2, 1743-1751. doi:10.1021/acsbiomaterials.6b00158

Park, J. W., Kang, Y. D., Kim, J. S., Lee, J. H., and Kim, H. W. (2014). 3D microenvironment of collagen hydrogel enhances the release of neurotrophic factors from human umbilical cord blood cells and stimulates the neurite outgrowth of human neural precursor cells. Biochem. Biophys. Res. Commun. 447, 400-406. doi:10.1016/j.bbrc.2014.03.145

Pham, Q. P., Sharma, U., and Mikos, A. G. (2006). Electrospinning of polymeric nanofibers for tissue engineering applications: a review. Tissue Eng. 12, 1197-1211. doi:10.1089/ten.2006.12.1197

Politis, M., and Lindvall, O. (2012). Clinical application of stem cell therapy in Parkinson's disease. BMC Med. 10:1. doi:10.1186/1741-7015-10-1

Pritchard, C. D., Slotkin, J. R., Yu, D., Dai, H., Lawrence, M. S., Bronson, R. T., et al. (2010). Establishing a model spinal cord injury in the African green monkey for the preclinical evaluation of biodegradable polymer scaffolds seeded with human neural stem cells. J. Neurosci. Methods 188, 258-269. doi:10.1016/ j.jneumeth.2010.02.019

Ratheesh, G., Venugopal, J. R., Chinappan, A., Ezhilarasu, H., Sadiq, A., and Ramakrishna, S. (2017). 3D fabrication of polymeric scaffolds for regenerative therapy. ACS Biomater. Sci. Eng. 3, 1175-1194. doi:10.1021/ acsbiomaterials.6b00370

Recknor, J. B., Recknor, J. C., Sakaguchi, D. S., and Mallapragada, S. K. (2004). Oriented astroglial cell growth on micropatterned polystyrene substrates. Biomaterials 25, 2753-2767. doi:10.1016/j.biomaterials.2003.11.045 
Sanders, J. E., Lamont, S. E., Karchin, A., Golledge, S. L., and Ratner, B. D. (2005). Fibro-porous meshes made from polyurethane micro-fibers: effects of surface charge on tissue response. Biomaterials 26, 813-818. doi:10.1016/ j.biomaterials.2004.03.030

Seaberg, R. M., and Van Der Kooy, D. (2003). Stem and progenitor cells: the premature desertion of rigorous definitions. Trends Neurosci. 26, 125-131. doi:10.1016/S0166-2236(03)00031-6

Seidlits, S. K., Schmidt, C. E., and Shear, J. B. (2009). High-resolution patterning of hydrogels in three dimensions using direct-write photofabrication for cell guidance. Adv. Funct. Mater. 19, 3543-3551. doi:10.1002/adfm.200901115

Shepherd, J. N. H., Parker, S. T., Shepherd, R. F., Gillette, M. U., Lewis, J. A., and Nuzzo, R. G. (2012). 3D microperiodic hydrogel scaffolds for robust neuronal cultures. Adv. Funct. Mater. 61, 47-54. doi:10.1002/adfm.201001746.3D

Shim,J. H., Kim,J. Y.,Park,M.,Park, J., andCho,D.W.(2011).Developmentofahybrid scaffold with synthetic biomaterials and hydrogel using solid freeform fabrication technology. Biofabrication 3, 034102. doi:10.1088/1758-5082/3/3/034102

Shin, Y., Yang, K., Han, S., Park, H. J., Heo, Y. S., Cho, S. W., et al. (2014). Reconstituting vascular microenvironment of neural stem cell niche in three-dimensional extracellular matrix. Adv. Healthc. Mater. 3, 1457-1464. doi:10.1002/adhm.201300569

Shrestha, B., Coykendall, K., Li, Y., Moon, A., Priyadarshani, P., and Yao, L. (2014). Repair of injured spinal cord using biomaterial scaffolds and stem cells. Stem Cell Res. Ther. 5, 91. doi:10.1186/scrt480

Siqueira, G., Kokkinis, D., Libanori, R., Hausmann, M. K., Gladman, A. S., Neels, A., et al. (2017). Cellulose nanocrystal inks for 3D printing of textured cellular architectures. Adv. Funct. Mater. 27:1604619. doi:10.1002/adfm.201604619

Smith, C. M., Stone, A. L., Parkhill, R. L., Stewart, R. L., Simpkins, M. W., Kachurin, A. M., et al. (2004). Three-dimensional bioassembly tool for generating viable tissue-engineered constructs. Tissue Eng. 10, 1566-1576. doi:10.1089/ten.2004. 10.1566

Stankus, J. J., Guan, J., Fujimoto, K., and Wagner, W. R. (2006). Microintegrating smooth muscle cells into a biodegradable elastomeric fiber matrix. Biomaterials 27, 735-744. doi:10.1002/nbm.3066

Thakur, A., Jaiswal, M. K., Peak, C. W., Carrow, J. K., Gentry, J., Dolatshahi-Pirouz, A., et al. (2016). Injectable shear-thinning nanoengineered hydrogels for stem cell delivery. Nanoscale 8, 12362-12372. doi:10.1039/C6NR02299E

Truby, R. L., and Lewis, J. A. (2016). Printing soft matter in three dimensions. Nature 540, 371-378. doi:10.1038/nature21003

Turunen, S., Haaparanta, A., Äänismaa, R., and Kellomäki, M. (2013). Chemical and topographical patterning of hydrogels for neural cell guidance in vitro. J. Tissue Eng. Regener. Med. 7, 253-270. doi:10.1002/term

Walker, C. L., Wang, X., Bullis, C., Liu, N.-K., Lu, Q., Fry, C., et al. (2015). Biphasic bisperoxovanadium administration and Schwann cell transplantation for repair after cervical contusive spinal cord injury. Exp. Neurol. 264, 163-172. doi:10.1016/j.expneurol.2014.12.002

Wang, T. Y., Bruggeman, K. F., Kauhausen, J. A., Rodriguez, A. L., Nisbet, D. R., and Parish,C.L. (2016). Functionalized composite scaffolds improve the engraftment of transplanted dopaminergic progenitors in a mouse model of Parkinson's disease. Biomaterials 74, 89-98. doi:10.1016/j.biomaterials.2015.09.039

Wang, X., Jiang, M., Zhou, Z., Gou, J., and Hui, D. (2017). 3D printing of polymer matrix composites: a review and prospective. Compos. Part B Eng. 110, 442-458. doi:10.1016/j.compositesb.2016.11.034

Weightman, A., Jenkins, S., Pickard, M., Chari, D., and Yang, Y. (2014). Alignment of multiple glial cell populations in 3D nanofiber scaffolds: toward the development of multicellular implantable scaffolds for repair of neural injury. Nanomedicine 10, 291-295. doi:10.1016/j.nano.2013.09.001

White,B.C.,Sullivan,J. M.,DeGracia,D.J., O’Neil,B.J.,Neumar,R.W., Grossman,L.I., et al. (2000). Brain ischemia and reperfusion: molecular mechanisms of neuronal injury. J. Neurol. Sci. 179, 1-33. doi:10.1016/S0022-510X(00) 00386-5

Xie, J., MacEwan, M. R., Willerth, S. M., Li, X., Moran, D. W., Sakiyama-Elbert, S. E., et al. (2009). Conductive core-sheath nanofibers and their potential application in neural tissue engineering. Adv. Funct. Mater. 19, 2312-2318. doi:10.1002/adfm.200801904

Yang, D., Lu, B., Zhao, Y., and Jiang, X. (2007). Fabrication of aligned fibrous arrays by magnetic electrospinning. Adv. Mater. Weinheim 19, 3702-3706. doi:10.1002/adma.200700171

Yao, S., Liu, X., Yu, S., Wang, X., Zhang, S., Wu, Q., et al. (2016). Co-effects of matrix low elasticity and aligned topography on stem cell neurogenic differentiation and rapid neurite outgrowth. Nanoscale 8, 10252-10265. doi:10.1039/ C6NR01169A

Yi, H., Choi, Y., Kang, K. S., Hong, J. M., Pati, R. G., Park, M. N., et al. (2016). A 3D-printed local drug delivery patch for pancreatic cancer growth suppression. J. Control. Release 238, 231-241. doi:10.1016/j.jconrel.2016.06.015

Yuan, Y.-M., and He, C. (2013). The glial scar in spinal cord injury and repair. Neurosci. Bull. 29, 421-435. doi:10.1007/s12264-013-1358-3

Zanatta, G., Steffens, D., Braghirolli, D. I., Fernandes, R. A., Netto, C. A., and Pranke, P. (2012). Viability of mesenchymal stem cells during electrospinning. Braz. J. Med. Biol. Res. 45, 125-130. doi:10.1590/S0100-879X2011007500163

Zhu, W., Harris, B. T., and Zhang, L. G. (2016). Gelatin methacrylamide hydrogel with graphene nanoplatelets for neural cell-laden 3D bioprinting. Conf. Proc. IEEE Eng. Med. Biol. Soc. 2016, 4185-4188. doi:10.1109/EMBC.2016. 7591649

Conflict of Interest Statement: The authors declare that the research was conducted in the absence of any commercial or financial relationships that could be construed as a potential conflict of interest.

Copyright (c) $2018 \mathrm{Meco}$ and Lampe. This is an open-access article distributed under the terms of the Creative Commons Attribution License (CC BY). The use, distribution or reproduction in other forums is permitted, provided the original author(s) and the copyright owner are credited and that the original publication in this journal is cited, in accordance with accepted academic practice. No use, distribution or reproduction is permitted which does not comply with these terms 